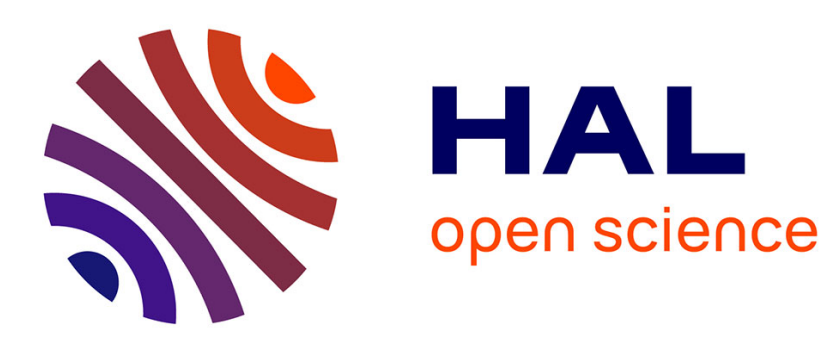

\title{
Stable Model Predictive Strategy for Rendezvous Hovering Phases Allowing for Control Saturation
}

\author{
Paulo Ricardo Arantes Gilz, Mioara Maria Joldes, Christophe Louembet, \\ Frédéric Camps
}

\section{- To cite this version:}

Paulo Ricardo Arantes Gilz, Mioara Maria Joldes, Christophe Louembet, Frédéric Camps. Stable Model Predictive Strategy for Rendezvous Hovering Phases Allowing for Control Saturation. Journal of Guidance, Control, and Dynamics, 2019, 42 (8), 10.2514/1.G003558 . hal-01678768v2

\section{HAL Id: hal-01678768 \\ https://hal.science/hal-01678768v2}

Submitted on 5 Nov 2018

HAL is a multi-disciplinary open access archive for the deposit and dissemination of scientific research documents, whether they are published or not. The documents may come from teaching and research institutions in France or abroad, or from public or private research centers.
L'archive ouverte pluridisciplinaire HAL, est destinée au dépôt et à la diffusion de documents scientifiques de niveau recherche, publiés ou non, émanant des établissements d'enseignement et de recherche français ou étrangers, des laboratoires publics ou privés. 


\title{
Stable Model Predictive Strategy for Rendezvous
}

\section{Hovering Phases Allowing for Control Saturation}

\author{
Paulo R. Arantes Gilz, Mioara Joldes ${ }^{\dagger}$ Christophe Louembet $\risingdotseq$ Frédéric Camps ${ }^{\S}$ \\ LAAS-CNRS, Université de Toulouse, CNRS, Toulouse, France
}

\begin{abstract}
This paper presents a model predictive control strategy for the spacecraft rendezvous hovering phases. Using a sequence of impulsive velocity changes, the spacecraft is controlled to reach and remain on a periodic trajectory inside a given box-type hover zone, while minimizing the fuel consumption. The path constraints (box-type and periodicity) are satisfied continuously in time, based on a particular parametrization of the linearized relative spacecraft trajectories. The control saturation constraint is enforced by re-planning. First, a sequence of saturated impulsive controls is selected such that the spacecraft gets on a periodic trajectory. Second, a fixed-length sequence of saturated impulses brings the spacecraft closer to the hover zone. The convergence of this approach is proved. Numerical methods are proposed to solve the required constrained optimization problems. Finally, hardware-in-the-loop simulations, using a synthesized LEON3 microprocessor, are performed to assess the efficiency and robustness of the proposed approach.
\end{abstract}

\section{Nomenclature}

$\|\cdot\|_{i} \quad \ell_{i}$-norm

| $\quad$ absolute value

$0_{n} \quad$ null matrix of dimension $n \times n$

$\tilde{A}(\nu), A_{D}(\nu)$ dynamics matrices for the states $\tilde{X}(\nu)$ and $D(\nu)$

a semi-major axis (m)

*PhD Graduate, Methods et Algorithms in Control, 7 Avenue du Colonel Roche; currently Université de Toulouse, Université Paul Sabatier, Laboratoire d'Analyse et d'Architectures des Systèmes, F-31400 Toulouse, France; prarante@laas .fr

${ }^{\dagger}$ Researcher, Methods et Algorithms in Control, Laboratoire d'Analyse et d'Architectures des Systèmes, LAAS-CNRS, 7 Avenue du Colonel Roche, F-31400 Toulouse, France; CNRS; joldes@laas.fr

$¥$ Associate Professor, Methods et Algorithms in Control, 7 Avenue du Colonel Roche; currently Université de Toulouse, Université Paul Sabatier, Laboratoire d'Analyse et d'Architectures des Systèmes, F-31400 Toulouse, France; louembet@laas.fr

${ }^{\S}$ Research Engineer, Methods et Algorithms in Control, 7 Avenue du Colonel Roche; currently Université de Toulouse, Université Paul Sabatier, Laboratoire d'Analyse et d'Architectures des Systèmes, F-31400 Toulouse, France; fcamps@laas.fr 


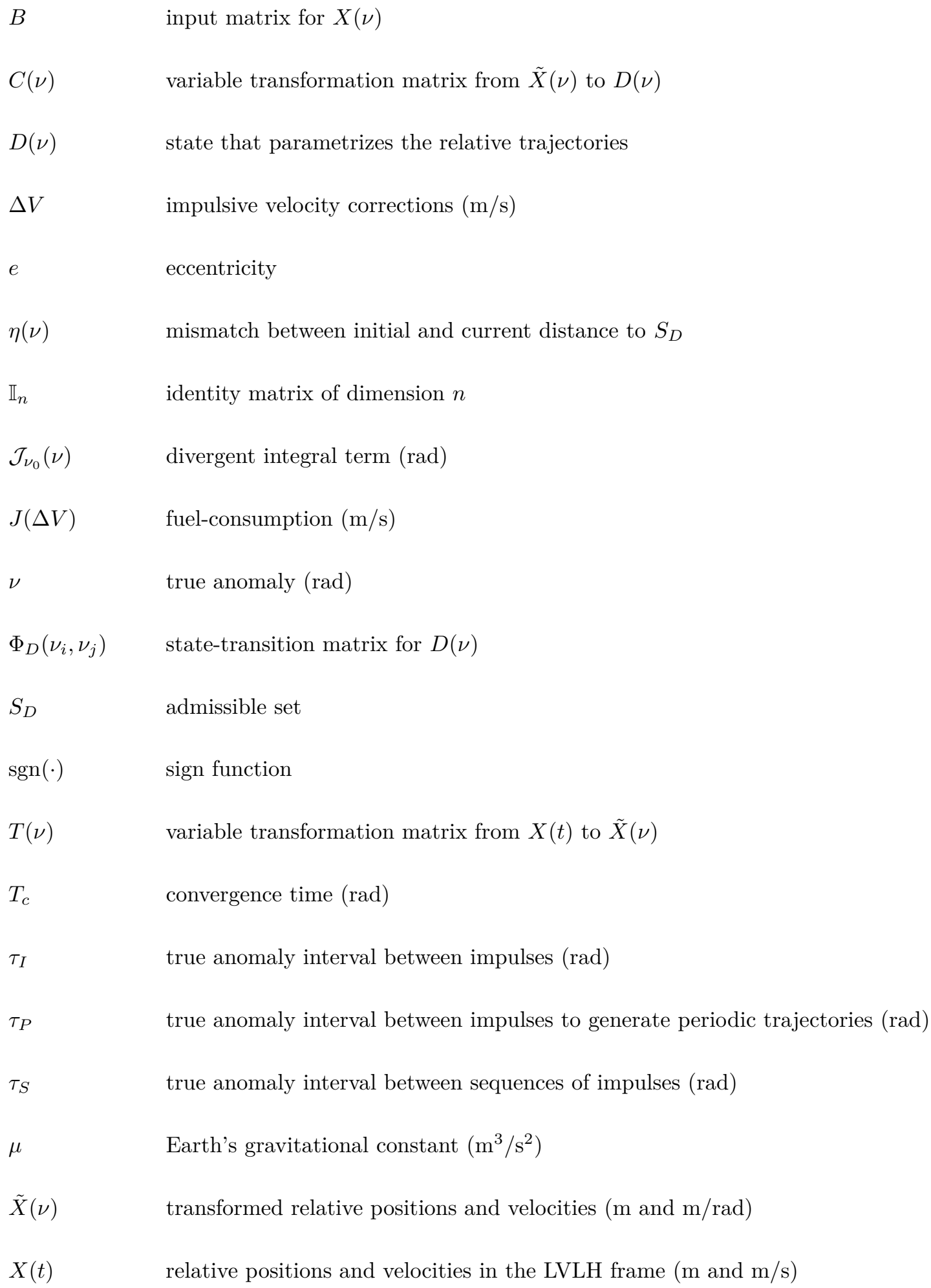

\section{Introduction}

Mastering the spacecraft rendezvous was crucial during the space conquest era and nowadays opens new economical opportunities for the space industry. In fact, numerous space tugs have been developed by the different actors in this industry. In particular, a key feature for mission success is the autonomy: 
the ability of the involved spacecraft to compute their guidance and control commands without human intervention.

The work presented in this paper aims to design a consumption-optimal controller compatible with the performance of a space flight certified computation board. This work is focused on the phases of the rendezvous problem in which a chaser spacecraft must hover inside a restricted tolerance zone in space (we assume a rectangular cuboid) defined in the local orbital frame of a leader spacecraft - these are the so-called "hovering" phases [1].

From the control point of view, the considered system is particular. First, the relative motion is governed by Keplerian dynamics. Second, it is controlled by means of chemical thrusters, which are able to provide high thrusts by mass ejection, but usually on a short time period. During the thrusting period, the relative velocity of the spacecraft evolves very quickly with respect to the Keplerian dynamics. Thus, the controlled system behavior can be represented by two dynamics with different time scales. Since the time scale difference is large, the rapid evolution of the relative velocity can be seen as an instantaneous change from the orbital dynamics perspective. Consequently, the control is considered as impulsive and the controlled relative motion is regarded as an impulsive system [2, chapter 1].

A wide range of control frameworks exist for handling these systems, among which adaptive control [3, 4], feedback impulsive control [5], Lyapunov-based non linear output feedback control [6], hybrid control [7, Chapter 1] and model predictive control.

Since the late nineties, model predictive control (MPC) schemes for impulsive spacecraft rendezvous have been developed with the aim of providing fuel-efficient and "flyable" control algorithms (cf. the recent review papers $[8,9])$. In fact, its popularity comes from the ability to account for constraints and uncertainties directly into the trajectory design thanks to the resolution of a constrained optimal control problem. The latter problem has also been extensively studied in the last two decades from different perspectives. On the one hand, the orbital elements have been exploited to design proximity maneuvers under separation constraints with the advantages of accounting for orbital disturbances in the dynamics $[10,11]$. On the other hand, the use of Cartesian elements also led to numerous works. First, the constrained optimal control problem has been converted into a linear program by means of dynamics discretization [12, 13]. Then, an analytic transition matrix (see for instance [14] and references therein) has been exploited to design impulsive maneuvers under the general Keplerian assumptions [15]. Different safety requirements have been addressed in several works such as visibility constraints [16], collision or plume avoidance [17, 18] or hovering [1]. In parallel, the effects of uncertainties on the open-loop, such as navigation errors or maneuver mis-execution, have also been studied [18-20].

The literature is rich of model predictive control applications for spacecraft rendezvous. In fact, model predictive control has been proven to ensure stability of the loop [21] even with the obstacle avoidance requirements [22]. It can also provide robustness to identified perturbations [23] or to navigation errors 
[24]. While the robust stability can be derived from a theoretical point of view [25], the features of such control schemes are lost if the recursive feasibility of the embedded programs can not be established. The constraint tightening $M P C$, permits to ensure the recursive feasibility by a priori tightening the constraints on the predicted states. The implementation of this technique necessitates either the existence of a stabilizing state-feedback controller [23, 26] or the off-line computation of some controls [27, 28]. Concerning the disturbances rejection, [29] implements techniques of tube-based MPC [30, 31] for fixedtime horizon rendezvous. With such an approach, the MPC controller avoids the open-loop propagation of the uncertainties and limits the spread of perturbed trajectories [32].

Most of the above cited works implement the predictive control theory in order to achieve a stable and robust closed loop. This fact often limits these approaches to linear time invariant Hill-ClohessyWiltshire dynamics [33] (see for instance [17, 21, 22, 24]).

If time-varying Tschauner-Hempel dynamics [34] are considered, conventional guidance approaches rely on constraints discretization for obtaining a tractable constrained optimization problem. A different approach was proposed in [35] by one of the authors of this article. The idea is to account for path constraints continuously in time, contrary to most techniques in literature, see $[15,16,18,23]$ and references therein. On the same lines, a preliminary result of the current work was presented in [36]. This article presented a new guidance law which exploits insight on the dynamics structure of the relative motion, like the periodicity of the Tschauner-Hempel dynamics and their analytic state transition matrix [14].

The main contribution of this work is to provide an MPC control algorithm, whose convergence is proved even if the hovering zone is unreachable because of control limitations. Our strategy aims at maintaining the chaser in a given window thanks to some periodic orbits which correspond to equilibrium points in the state space. A similar idea was proposed in [37] for different linear impulsive systems, in the biomedical field.

The stability result is obtained by first discarding the saturation constraint from the constrained optimal control problem, in order to ensure the recursive feasibility, and then scaling the obtained control impulses, if they exceed the saturation threshold. Finally, we prove that employing a certain sequence of such impulses ensures the convergence to (and the invariance with respect to) the hovering zone.

In another related work [38], this problem is handled by considering impulsive systems as part of hybrid systems [7, Chapter 1]. This resulted in several hybrid control laws, for which the ability to stabilize the chaser vehicle around a given relative orbit was proven. However, this approach is not able to account for any constraints on the state or command. Those hybrid controllers are implemented to assess the relevance of the proposed predictive controller by means of comparisons.

Finally, an important contribution consists also in performing simulations in a hardware-in-the-loop 
fashion, following the framework of the tests carried out in [39, 40]. The control is computed on a synthesized FPGA LEON3 board certified for spacecraft usage, while the space environment is simulated on a high fidelity simulator [41]. The performances of the predictive controller are assessed by means of non linear simulations featuring orbital disturbances, navigation and control execution errors.

In parallel with this work, the numerical efficiency of the programming instances on which the predictive controller relies on, has been assessed in terms of numerical burden, by means of extensive runs and compared to the classical scheme based on linear programming [42]. This study shows that accounting for path constraints continuously in time (as in the proposed approach) and solving the discretizedconstraints instances by means linear programming, are both equivalent in terms of computation burden and footprint. This strengthens the practical pertinence of the proposed method, since it has the advantage of avoiding the systematic constraints violation due to discretization.

\section{Modeling the rendezvous hovering phases problem}

In this section we present the assumptions and equations employed in the modeling of the linearized relative dynamics between spacecraft. Once this model is obtained, a parametrization of the periodic space-constrained relative trajectories is deduced by performing transformations of the state variable. These developments are employed in Section 3 for the formulation of the MPC scheme and its stability proof, as well as in Section 2.7 for the conception of semi-algebraic check functions for the space constraints.

\subsection{Relative spacecraft motion}

Figure 1 presents the frames used to model the relative motion between the leader $S_{l}$ and the follower $S_{f}$ spacecrafts. The Earth-Centered Inertial and the Local Vertical / Local Horizontal (LVLH) frames are respectively given by $\{O, \vec{I}, \vec{J}, \vec{K}\}$ and $\left\{S_{l}, \vec{x}, \vec{y}, \vec{z}\right\}$.

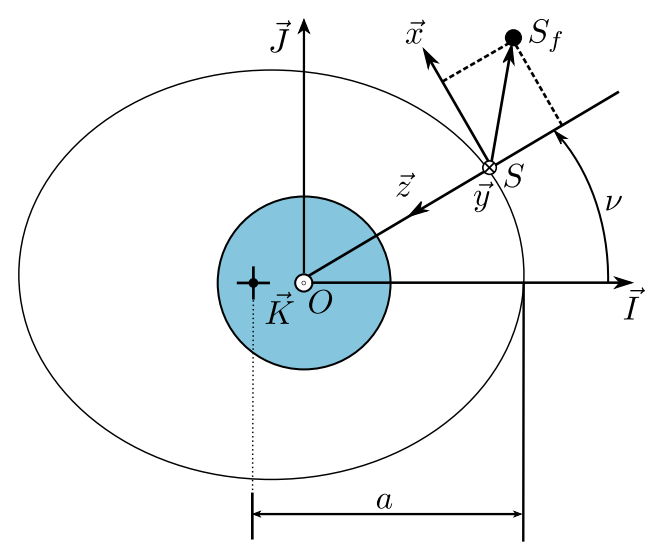

Figure 1: Inertial and relative frames

The angle $\nu$, between the direction of perigee and the leader's position, is the true anomaly, $0<e<1$ 
is the leader orbit eccentricity, $a$ is the leader orbit semi-major axis and $\mu$ is Earth's gravitational constant. Considering that $\left\|\overrightarrow{O S_{l}}\right\| \gg\left\|\overrightarrow{S_{l} S_{f}}\right\|$ and assuming Keplerian orbits, the relative dynamics can be linearized and described by the simplified linearized Tschauner-Hempel equations [34]:

$$
\tilde{X}_{x z}^{\prime}(\nu)=\underbrace{\left[\begin{array}{cccc}
0 & 0 & 1 & 0 \\
0 & 0 & 0 & 1 \\
0 & 0 & 0 & 2 \\
0 & \frac{3}{\rho_{\nu}} & -2 & 0
\end{array}\right]}_{\tilde{A}_{x z}(\nu)} \tilde{X}_{x z}(\nu), \quad \tilde{X}_{y}^{\prime}(\nu)=\underbrace{\left[\begin{array}{cc}
0 & 1 \\
-1 & 0
\end{array}\right]}_{\tilde{A}_{y}(\nu)} \tilde{X}_{y}(\nu),
$$

where $(\cdot)^{\prime}=\frac{d(\cdot)}{d \nu}$ and $\rho_{\nu}=\rho(\nu)=(1+e \cos \nu)$.

In (1), we explicitly split the equations to emphasize the fact that the so-called in-plane motion ( $x z$-plane) is decoupled from the out-of-plane motion (y-axis). The independent variable is the true anomaly $\nu$, since this often results in simplified computations. In particular, a closed-form transition matrix $\Phi\left(\nu, \nu_{0}\right)$, which describes the solution of equation (1) was proposed in [14]:

$$
\tilde{X}(\nu)=\Phi\left(\nu, \nu_{0}\right) \tilde{X}\left(\nu_{0}\right), \quad \forall \nu \geq \nu_{0}
$$

For a given time $t$, the change of variable from the LVLH state $X(t)=[x(t), y(t), z(t), \dot{x}(t), \dot{y}(t), \dot{z}(t)]^{T}$ to the $\tilde{X}(\nu)$ vector can be performed via the following transformation:

$$
\tilde{X}(\nu)=T(\nu) X(t), \quad \text { with } \quad T(\nu)=\left[\begin{array}{cc}
\rho_{\nu} \mathbb{I}_{3} & 0_{3} \\
\rho_{\nu}^{\prime} \mathbb{I}_{3} & \left(k^{2} \rho_{\nu}\right)^{-1} \mathbb{I}_{3}
\end{array}\right],
$$

where $k^{2}=\sqrt{\frac{\mu}{a^{3}\left(1-e^{2}\right)^{3}}}$.

During the rendezvous hovering phases, the follower spacecraft is required to remain in the interior of a certain limited region of the space. We assume in the sequel that this hovering range is a rectangular cuboid (tolerance box), in the LVLH frame:

$$
\underline{x} \leq x(t) \leq \bar{x} \quad \underline{y} \leq y(t) \leq \bar{y} \quad \underline{z} \leq z(t) \leq \bar{z}, \quad \forall t \geq t_{0} .
$$

In order to obtain a better-suited representation of these constrained relative trajectories, another variable change $[35,43]$ is performed, which defines the so-called vector of parameters:

$$
D(\nu)=\left[d_{0}(\nu), d_{1}(\nu), d_{2}(\nu), d_{3}(\nu), d_{4}(\nu), d_{5}(\nu)\right]^{T} .
$$

The idea is to obtain parametric expressions for the spacecraft relative positions by expanding the terms 
in equation (2) and then factoring out the terms related to the independent variable $\nu$ :

$$
\begin{aligned}
& \tilde{x}(\nu)=d_{1}\left(\nu_{0}\right)\left(2+e c_{\nu}\right) s_{\nu}-d_{2}\left(\nu_{0}\right)\left(2+e c_{\nu}\right) c_{\nu}+d_{3}\left(\nu_{0}\right)+3\left(1+e c_{\nu}\right)^{2} d_{0}\left(\nu_{0}\right) \mathcal{J}_{\nu_{0}}(\nu), \\
& \tilde{y}(\nu)=d_{4}\left(\nu_{0}\right) c_{\nu}+d_{5}\left(\nu_{0}\right) s_{\nu}, \\
& \tilde{z}(\nu)=d_{1}\left(\nu_{0}\right)\left(1+e c_{\nu}\right) c_{\nu}+d_{2}\left(\nu_{0}\right)\left(1+e c_{\nu}\right) s_{\nu}-3 e s_{\nu}\left(1+e c_{\nu}\right) d_{0}\left(\nu_{0}\right) \mathcal{J}_{\nu_{0}}(\nu)+2 d_{0}\left(\nu_{0}\right),
\end{aligned}
$$

where $s_{\nu}=\sin (\nu), c_{\nu}=\cos (\nu)$ and $\mathcal{J}_{\nu_{0}}(\nu)$ is the only nonperiodic term, given by:

$$
\mathcal{J}_{\nu_{0}}(\nu):=\int_{\nu_{0}}^{\nu} \frac{d \tau}{\rho(\tau)^{2}}=\left[\frac{2 e \tan \left(\frac{x}{2}\right)}{\left(e^{2}-1\right)\left((1-e) \tan \left(\frac{x}{2}\right)^{2}+e+1\right)}-\frac{2 \operatorname{arctanh}\left(\frac{\tan \left(\frac{x}{2}\right) \sqrt{e-1}}{\sqrt{e+1}}\right)}{\left(e^{2}-1\right)^{\frac{3}{2}}}\right]_{\nu_{0}}^{\nu} .
$$

This integral term also has a close-form expression with respect to the elapsed time $t-t_{0}$ in seconds,

$$
\mathcal{J}_{\nu_{0}}(\nu):=\sqrt{\frac{\mu}{a^{3}}} \frac{t-t_{0}}{\left(1-e^{2}\right)^{3 / 2}} .
$$

The main advantage of the parametric expression (6) is that a periodic relative trajectory can be obtained by requiring the parameter $d_{0}$ to be zero, which cancels the effect of the drifting term $\mathcal{J}_{\nu_{0}}(\nu)$. The other parameters also provide some physical description of the relative orbits (e.g., $d_{3}$ represents a constant offset on the $x$ axis), but a formal link between their values and the shape of the resulting spacecraft relative trajectory is not easy to establish.

Analogously, $D(\nu)$ can also be split into two vectors representing respectively the in-plane and the out-of-plane motion: $D_{x z}(\nu)=\left[d_{0}(\nu), d_{1}(\nu), d_{2}(\nu), d_{3}(\nu)\right]^{T}$ and $D_{y}(\nu)=\left[d_{4}(\nu), d_{5}(\nu)\right]^{T}$.

This state can be expressed in function of $\tilde{X}$ as follows:

$$
D_{x z}(\nu)=\underbrace{\frac{1}{e^{2}-1}\left[\begin{array}{cccc}
0 & -\left(3 e c_{\nu}+e^{2}+2\right) & \rho_{\nu}^{2} & -e s_{\nu} \rho_{\nu} \\
0 & 3\left(e+c_{\nu}\right) & -\left(2 c_{\nu}+e c_{\nu}^{2}+e\right) & s_{\nu} \rho_{\nu} \\
0 & \frac{3 s_{\nu}\left(\rho_{\nu}+e^{2}\right)}{\rho_{\nu}} & -s_{\nu}\left(2+e c_{\nu}\right) & 2 e-c_{\nu} \rho_{\nu} \\
1 & \frac{-3 e s_{\nu}\left(2+e c_{\nu}\right)}{\rho_{\nu}} & e s_{\nu}\left(2+e c_{\nu}\right) & e c_{\nu} \rho_{\nu}-2
\end{array}\right]}_{C_{x z}(\nu)} \tilde{X}_{x z}(\nu), \quad D_{y}(\nu)=\underbrace{\left[\begin{array}{cc}
c_{\nu} & -s_{\nu} \\
s_{\nu} & c_{\nu}
\end{array}\right]}_{C_{y}(\nu)} \tilde{X}_{y}(\nu) .
$$

A concise form of (9) is given by $D(\nu)=C(\nu) \tilde{X}(\nu)$. By noting that

$$
\operatorname{det}(C(\nu))=\frac{1}{1-e^{2}} \neq 0, \text { for } 0 \leq e<1,
$$

one can conclude that matrix $C(\nu)$ defines a similar transformation and $D$ is a proper state vector.

By manipulating equations (1) and (9) (see [43] for details), one can obtain the following dynamical 
system representing the evolution of state $D$ :

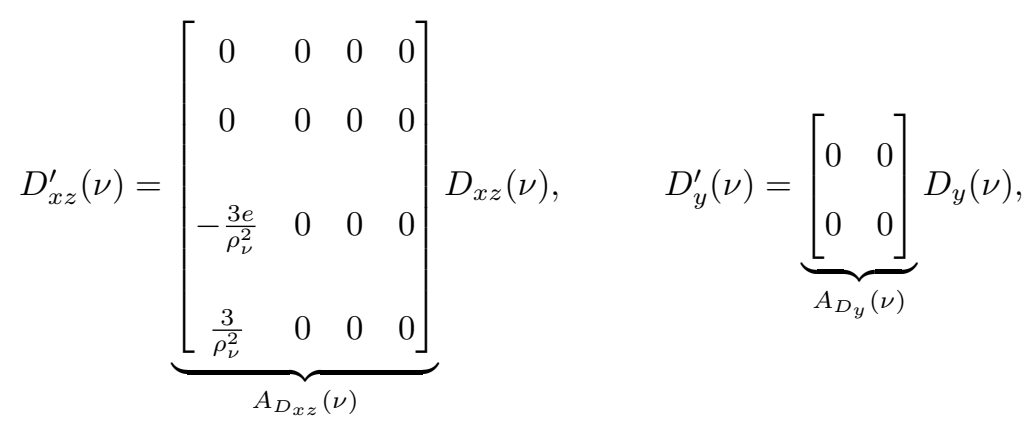

which can also be described by its transition matrix:

$$
D_{x z}(\nu)=\underbrace{\left[\begin{array}{cccc}
1 & 0 & 0 & 0 \\
0 & 1 & 0 & 0 \\
-3 e \mathcal{J}_{\nu_{0}}(\nu) & 0 & 1 & 0 \\
3 \mathcal{J}_{\nu_{0}}(\nu) & 0 & 0 & 1
\end{array}\right]}_{\Phi_{D_{x z}}\left(\nu, \nu_{0}\right)} D_{x z}\left(\nu_{0}\right), \quad D_{y}(\nu)=\underbrace{\left[\begin{array}{cc}
1 & 0 \\
0 & 1
\end{array}\right]}_{\Phi_{D_{y}}\left(\nu, \nu_{0}\right)} D_{y}\left(\nu_{0}\right) .
$$

Remark 2.1. Hereafter we use the notation $C(\nu), A_{D}(\nu)$ and $\Phi_{D}\left(\nu_{N}, \nu_{i}\right)$ to refer to the concatenation of the respective in-plane and out-of-plane matrices.

\subsection{Impulsive control framework}

In this work, impulsive control of the relative motion is considered. In fact, the chaser spacecraft is moved by chemical engines that provide high level of thrust during a short time with respect to the target orbital period. This leads to an extremely rapid change of velocity, which can be modeled as a jump in the velocity history at time $t_{i}$ :

$$
X^{+}\left(t_{i}\right)=X\left(t_{i}\right)+B \Delta V\left(t_{i}\right)
$$

where $X^{+}\left(t_{i}\right)=\lim _{t \rightarrow t_{i}^{+}} X(t), B=\left[0_{3} \mathbb{I}_{3}\right]$ and $\Delta V\left(t_{i}\right)=\left[\Delta V_{x}\left(t_{i}\right), \Delta V_{y}\left(t_{i}\right), \Delta V_{z}\left(t_{i}\right)\right]^{T}$. Applying the changes of variable (3) and (9), an impulse at time $\nu_{i}$ produces a jump in the $D$ state trajectory:

$$
D^{+}\left(\nu_{i}\right)=D\left(\nu_{i}\right)+\underbrace{C\left(\nu_{i}\right) T\left(\nu_{i}\right) B}_{B_{D}} \Delta V\left(\nu_{i}\right)
$$

where $D^{+}\left(\nu_{i}\right)=\lim _{\nu \rightarrow \nu_{i}^{+}} D(\nu)$. Equation (13) also shows that a given impulsive control $\Delta V$ will have a different impact, depending on the time of application, due to the time dependence of the input matrix $B_{D}$

Finally, the controlled system can be described following the impulsive system formalism presented 
in [7, chapter 1]. This consists of: (i) a continuous-time differential equation that governs the motion between impulses; (ii) a difference equation that states the instantaneous change when an impulsive control occurs; (iii) a prescribed sequence of impulsive times:

$$
\begin{cases}D^{\prime}(\nu)=A_{D}(\nu) D(\nu) & \nu \neq \nu_{i}, i=1,2, \ldots \\ D^{+}\left(\nu_{i}\right)=D\left(\nu_{i}\right)+B_{D}\left(\nu_{i}\right) \Delta V\left(\nu_{i}\right) & \nu=\nu_{i}, i=1,2, \ldots\end{cases}
$$

Note that, if the impulsive system framework in the sense of [2, chapter 1] is adopted, the system (14) can be viewed as a special case in the more general frame of hybrid dynamical systems [7]. Moreover, the analytic transition matrix of the system (11) is exploited in the design of the model predictive controller. Specifically, the state $D$ right after a given a sequence of $N$ impulses applied at $\nu_{1} \ldots \nu_{N-1}, \nu_{N}$, becomes:

$$
D^{+}\left(\nu_{N}\right)=\Phi_{D}\left(\nu_{N}, \nu_{1}\right) D\left(\nu_{1}\right)+\sum_{i=1}^{N} \underbrace{\Phi_{D}\left(\nu_{N}, \nu_{i}\right) C\left(\nu_{i}\right) T\left(\nu_{i}\right) B}_{B_{D}\left(\nu_{N}, \nu_{i}\right)} \Delta V\left(\nu_{i}\right),
$$

or, similarly, by splitting the dynamics into out-of-plane and in-plane motion, we obtain:

$$
\begin{aligned}
& D_{x z}^{+}\left(\nu_{N}\right)=\Phi_{D_{x z}}\left(\nu_{N}, \nu_{1}\right) D_{x z}\left(\nu_{1}\right)+\sum_{i=1}^{N} B_{D_{x z}}\left(\nu_{N}, \nu_{i}\right) \Delta V_{x z}\left(\nu_{i}\right) \\
& D_{y}^{+}\left(\nu_{N}\right)=\Phi_{D_{y}}\left(\nu_{N}, \nu_{1}\right) D_{y}\left(\nu_{1}\right)+\sum_{i=1}^{N} B_{D_{y}}\left(\nu_{N}, \nu_{i}\right) \Delta V_{y}\left(\nu_{i}\right)
\end{aligned}
$$

where $\Delta V_{x z}\left(\nu_{i}\right)=\left[\Delta V_{x}\left(\nu_{i}\right), \Delta V_{z}\left(\nu_{i}\right)\right]^{T}$,

$$
\begin{aligned}
& B_{D_{x z}}\left(\nu_{N}, \nu\right)=\frac{1}{k^{2}\left(e^{2}-1\right) \rho_{\nu}}\left[\begin{array}{cc}
\rho_{\nu}^{2} & -e s_{\nu} \rho_{\nu} \\
-\left(2 c_{\nu}+e c_{\nu}^{2}+e\right) & s_{\nu} \rho_{\nu} \\
-s_{\nu}\left(2+e c_{\nu}\right)-3 e \mathcal{J}_{\nu}\left(\nu_{N}\right) & 2 e-c_{\nu} \rho_{\nu}+3 e^{2} s_{\nu} \rho_{\nu} \mathcal{J}_{\nu}\left(\nu_{N}\right) \\
e s_{\nu}\left(2+e c_{\nu}\right)+3 \mathcal{J}_{\nu}\left(\nu_{N}\right) & e c_{\nu} \rho_{\nu}-2-3 e s_{\nu} \rho_{\nu} \mathcal{J}_{\nu}\left(\nu_{N}\right)
\end{array}\right] \\
& B_{D_{y}}\left(\nu_{N}, \nu\right)=\frac{1}{k^{2} \rho_{\nu}}\left[\begin{array}{c}
-s_{\nu} \\
c_{\nu}
\end{array}\right] .
\end{aligned}
$$

By introducing the matrices:

$$
\begin{aligned}
& M\left(\nu_{1}, \ldots, \nu_{N}\right):=\left[B_{D}\left(\nu_{N}, \nu_{1}\right), \ldots, B_{D}\left(\nu_{N}, \nu_{N}\right)\right] \\
& M_{x z}\left(\nu_{1}, \ldots, \nu_{N}\right):=\left[B_{D_{x z}}\left(\nu_{N}, \nu_{1}\right), \ldots, B_{D_{x z}}\left(\nu_{N}, \nu_{N}\right)\right] \\
& M_{y}\left(\nu_{1}, \ldots, \nu_{N}\right):=\left[B_{D_{y}}\left(\nu_{N}, \nu_{1}\right), \ldots, B_{D_{y}}\left(\nu_{N}, \nu_{N}\right)\right]
\end{aligned}
$$


and the vectors:

$$
\begin{aligned}
& \Delta V:=\left[\begin{array}{lll}
\Delta V\left(\nu_{1}\right), & \ldots & \Delta V\left(\nu_{N}\right)
\end{array}\right]^{T}, \\
& \Delta V_{x z}:=\left[\begin{array}{lllll}
\Delta V_{x}\left(\nu_{1}\right), & \Delta V_{z}\left(\nu_{1}\right) & \ldots & \Delta V_{x}\left(\nu_{N}\right), & \Delta V_{z}\left(\nu_{N}\right)
\end{array}\right]^{T} \text {, } \\
& \Delta V_{y}:=\left[\begin{array}{lll}
\Delta V_{y}\left(\nu_{1}\right), & \ldots & \Delta V_{y}\left(\nu_{N}\right)
\end{array}\right]^{T},
\end{aligned}
$$

expressions (15) and (16) can be further simplified:

$$
\begin{aligned}
& D^{+}\left(\nu_{N}\right)=\Phi_{D}\left(\nu_{N}, \nu_{1}\right) D\left(\nu_{1}\right)+M\left(\nu_{1}, \ldots, \nu_{N}\right) \Delta V, \\
& D_{x z}^{+}\left(\nu_{N}\right)=\Phi_{D_{x z}}\left(\nu_{N}, \nu_{1}\right) D_{x z}\left(\nu_{1}\right)+M_{x z}\left(\nu_{1}, \ldots, \nu_{N}\right) \Delta V_{x z}, \\
& D_{y}^{+}\left(\nu_{N}\right)=\Phi_{D_{y}}\left(\nu_{N}, \nu_{1}\right) D_{y}\left(\nu_{1}\right)+M_{y}\left(\nu_{1}, \ldots, \nu_{N}\right) \Delta V_{y} .
\end{aligned}
$$

In what follows, the problem constraints are presented. They intervene in the constrained fuel-optimal control problem that is to be solved at each call of the model predictive controller.

\subsection{Thrusters, fuel-consumption and saturation}

We suppose that the follower spacecraft has six identical thrusters, one pair symmetrically and oppositely disposed by axis, as indicated in Fig. 2 .

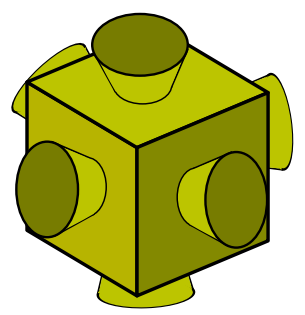

Figure 2: Thrusters arrangement

The fuel consumption for $N$ instantaneous impulsive velocity corrections is then modeled as the sum of the absolute value of the thrusts applied in each direction:

$$
J(\Delta V)=\sum_{i=1}^{N}\left\|\Delta V\left(\nu_{i}\right)\right\|_{1}=\sum_{i=1}^{N}\left|\Delta V_{x}\left(\nu_{i}\right)\right|+\left|\Delta V_{y}\left(\nu_{i}\right)\right|+\left|\Delta V_{z}\left(\nu_{i}\right)\right|,
$$

and assuming that the saturation threshold for each thruster is $\overline{\Delta V}>0$, this constraint is written as:

$$
\left|\Delta V_{x}\left(\nu_{i}\right)\right| \leq \overline{\Delta V}, \quad\left|\Delta V_{y}\left(\nu_{i}\right)\right| \leq \overline{\Delta V}, \quad\left|\Delta V_{z}\left(\nu_{i}\right)\right| \leq \overline{\Delta V} .
$$




\subsection{Space constrained periodic relative trajectories}

One is interested in obtaining periodic trajectories that is, $\tilde{X}(\nu+2 \pi)=\tilde{X}(\nu), \forall \nu$, since this guarantees that the relative motion remains bounded in absence of disturbances. Since the only non-periodic and divergent term $\mathcal{J}_{\nu_{0}}(\nu)$ in the relative motion equations (6) always appears multiplied by $d_{0}$, one can deduce that a necessary and sufficient condition for this constraint to be satisfied is:

$$
d_{0}\left(\nu_{0}\right)=0 .
$$

Remark 2.2. Periodic trajectories produce no drift. A notable property of periodic trajectories is that the corresponding state vector $D(\nu)$ remains constant for any value of $\nu$ since its dynamic matrix $A_{D}(\nu)$ has non-zero values only in its first column (see (10)).

By imposing $d_{0}=0$ (periodicity condition) and using (3) and (9), the inequalities in (4) are rewritten as:

$$
\begin{aligned}
& \underline{x} \leq F_{x}(\nu)^{T} D_{x z} \leq \bar{x} \quad F_{x}(\nu)=\left[\quad 0, \frac{\left(2+e c_{\nu}\right) s_{\nu}}{1+e c_{\nu}}, \quad \frac{-\left(2+e c_{\nu}\right) c_{\nu}}{1+e c_{\nu}}, \frac{1}{1+e c_{\nu}}\right]^{T} \\
& \underline{y} \leq F_{y}(\nu)^{T} D_{y} \leq \bar{y}, \forall \nu, \quad F_{z}(\nu)=\left[\begin{array}{llllll}
0, & c_{\nu}, & s_{\nu}, & 0
\end{array}\right]^{T} \\
& \underline{z} \leq F_{z}(\nu)^{T} D_{x z} \leq \bar{z} \quad F_{y}(\nu)=\left[\begin{array}{ccc}
\frac{c_{\nu}}{1+e c_{\nu}}, & \frac{s_{\nu}}{1+e c_{\nu}}
\end{array}\right]^{T}
\end{aligned}
$$

The admissible set i.e. the set of states $D$ (points in $\mathbb{R}^{6}$ ) that correspond to periodic relative trajectories satisfying the inequalities in (22), is then defined as:

$$
S_{D}:=\left\{D \in \mathbb{R}^{6} \mid \begin{array}{c}
\underline{x} \leq F_{x}(\nu)^{T} D_{x z} \leq \bar{x} \\
d_{0}=0, \\
\underline{y} \leq F_{y}(\nu)^{T} D_{y} \leq \bar{y} \quad, \forall \nu \\
\underline{z} \leq F_{z}(\nu)^{T} D_{x z} \leq \bar{z}
\end{array}\right\}
$$

and since the out-of-plane and in-plane dynamics can be decoupled, we also define:

$$
\begin{aligned}
S_{D_{y}} & =\left\{D_{y} \in \mathbb{R}^{2} \mid \underline{y} \leq F_{y}(\nu)^{T} D_{y} \leq \bar{y}, \forall \nu\right\}, \\
S_{D_{x z}} & =\left\{D_{x z} \in \mathbb{R}^{4} \mid \begin{array}{l}
d_{0}=0, \\
\underline{x} \leq F_{x}(\nu)^{T} D_{x z} \leq \bar{x} \\
\underline{z} \leq F_{z}(\nu)^{T} D_{x z} \leq \bar{z}
\end{array}, \forall \nu\right\} .
\end{aligned}
$$

Proposition 2.1. The admissible set $S_{D}$ is compact and convex.

Proof. The detailed proof is presented in Appendix A.1.

The description of the admissible set previously presented consists in the evaluation of infinitely many affine inequalities on the state $D$, which depends on $\nu$. Although theoretically interesting, this 
description is not practical from a numerical point of view, since the infinitely many constraints are hard to be accounted for, when employed in the formulation of optimization problems. In Sections 2.6 and 2.7 , alternative finite descriptions ${ }^{1}$ are presented.

\subsection{Fuel-optimal impulsive control problem}

Once the mathematical models for the evolution of the $D$ state and for the constraints are obtained (Sections 2.3 and 2.4), we can formulate the fuel-optimal impulsive control problem that is solved at each call of the MPC algorithm:

Problem 1. Given $N \geq 3, \nu_{1}<\ldots<\nu_{N} \in \mathbb{R}_{>0}, D\left(\nu_{1}\right) \in \mathbb{R}^{6}$, $e \in \mathbb{R}_{[0,1]}, a, \mu \in \mathbb{R}_{>0}$, find $\Delta V^{*} \in \mathbb{R}^{3 N}$ such that:

$$
\begin{array}{cl}
\Delta V^{*}=\underset{\Delta V}{\operatorname{argmin}} & J(\Delta V) \\
\text { s.t. } & \left\{\begin{array}{l}
D\left(\nu_{1}\right)=D_{1} \\
D^{+}\left(\nu_{N}\right)=\Phi_{D}\left(\nu_{N}, \nu_{1}\right) D\left(\nu_{1}\right)+M\left(\nu_{1}, \ldots, \nu_{N}\right) \Delta V \\
D^{+}\left(\nu_{N}\right) \in S_{D}
\end{array}\right.
\end{array}
$$

Problem $\mathcal{P}$ accounts for both the in-plane and out-of-plane dynamics, even though they can be solved separately (in Section 3, these dynamics are split and represented by the functions $\gamma_{x z}$ and $\gamma_{y}$ respectively). Moreover, one can remark that the control actions are not saturated in the formulation of Problem $\mathcal{P}$; an ad hoc function $\gamma_{\overline{\Delta V}}$ is employed to take these constraints into account in Section 3.

Hereafter, we present two different finite descriptions for the admissible set and the respective methods employed on the resolution of their associated fuel-optimal impulsive optimization problems.

\subsection{Semi-definite programming description of periodic space-constrained rel- ative trajectories}

A finite mathematical description of the periodic relative trajectories respecting polytopic space constraints is presented in $[29,35,43]$. This description is obtained by performing a variable change that converts each one of the "belonging to one side of the plane" constraints into one polynomial non-negative constraint; then a certificate of positivity demonstrated by Nesterov in [44] is applied. This result links the univariate polynomial non-negativity to the existence of a semi-definite positive matrix whose entries are related to the coefficients of the polynomial. By performing variable changes, the space-constraints inequalities in (4) can then be reformulated as:

$$
\Gamma_{\underline{x}}(s) \geq 0, \quad \Gamma_{\bar{x}}(s) \geq 0, \quad \Gamma_{\underline{y}}(s) \geq 0, \quad \Gamma_{\bar{y}}(s) \geq 0, \quad \Gamma_{\underline{z}}(s) \geq 0, \quad \Gamma_{\bar{z}}(s) \geq 0, \quad \forall s \in \mathbb{R} .
$$

\footnotetext{
${ }^{1}$ descriptions in which a finite number of free variables satisfy a finite number of constraints
} 
Let $\gamma_{w}$ be the vector of coefficients of the polynomial $\Gamma_{w}$ in (26), where $w$ replaces the upper and lower bounds $\{\underline{x}, \bar{x}, \underline{y}, \bar{y}, \underline{z}, \bar{z}\}$. Applying Nesterov results [44, Theorem 17.10], we finally obtain the following description for the admissible set:

$$
S_{D}=\left\{D \in \mathbb{R}^{6} \mid d_{0}=0, \exists Y_{w} \succeq 0 \text { s.t } \gamma_{w}=\Lambda^{*}\left(Y_{w}\right), w \in\{\underline{x}, \bar{x}, \underline{y}, \bar{y}, \underline{z}, \bar{z}\}\right\}
$$

where the operator $\Lambda^{*}$ is defined by:

$$
\Lambda^{*}(Y)(j)=\operatorname{tr}\left(Y H_{m, j}\right), \quad j=1, \ldots, 2 m+1
$$

and $H_{m, j} \in \mathbb{R}^{(m+1) \times(m+1)}$ are the Hankel matrices that contain ones on the $j$-th anti-diagonal and zeros elsewhere. The associated fuel-optimal impulsive optimization problem is formulated as follows:

Problem 2. Given $N \geq 3, \nu_{1}<\ldots<\nu_{N} \in \mathbb{R}_{>0}, D\left(\nu_{1}\right) \in \mathbb{R}^{6}, e \in \mathbb{R}_{[0,1]}, a, \mu \in \mathbb{R}_{>0}$, find $\Delta V^{*} \in \mathbb{R}^{3 N}$ such that:

$$
\begin{array}{cl}
\Delta V^{*}=\underset{\Delta V}{\operatorname{argmin}} & J(\Delta V) \\
\text { s.t. } & \left\{\begin{array}{l}
D\left(\nu_{1}\right)=D_{1} \\
D^{+}\left(\nu_{N}\right)=\Phi_{D}\left(\nu_{N}, \nu_{1}\right) D\left(\nu_{1}\right)+M\left(\nu_{1}, \ldots, \nu_{N}\right) \Delta V \\
d_{0}^{+}\left(\nu_{N}\right)=0 \\
\exists Y_{w} \succeq 0 \text { s.t. } \gamma_{w}\left(D^{+}\left(\nu_{N}\right)\right)=\Lambda^{*}\left(Y_{w}\right), w \in\{\underline{x}, \bar{x}, \underline{y}, \bar{y}, \underline{z}, \bar{z}\}
\end{array}\right.
\end{array}
$$

This problem can be solved by dedicated semi-definite program (SDP) solvers (SDPA, SeDuMi, SDPT3, CSDP, etc).

\subsection{Envelope description of periodic space-constrained relative trajectories}

In [36] a redefinition of the set of states corresponding to periodic space-constrained trajectories is provided. This consists in finding the envelope of the curves which define the boundary of the admissible set, which boils down to the evaluation of several convex semi-algebraic functions. Therein, the periodic vectors of parameters $D \in \mathbb{R}^{6}$, s.t. $d_{0}=0$ respecting the space-constraints inequalities (4) are such that:

$$
g_{\underline{x}}(D) \leq 0, \quad g_{\bar{x}}(D) \leq 0, \quad g_{\underline{y}}(D) \leq 0, \quad g_{\bar{y}}(D) \leq 0, \quad g_{\underline{z}}(D) \leq 0, \quad g_{\bar{z}}(D) \leq 0,
$$

where the functions $g_{w}(D)$ are convex but non-differentiable functions. Hence the admissible set can be redefined as:

$$
S_{D}=\left\{D \in \mathbb{R}^{6} \mid d_{0}=0, g_{w}(D) \leq 0, w \in\{\underline{x}, \bar{x}, \underline{y}, \bar{y}, \underline{z}, \bar{z}\}\right\}
$$

and the associated fuel-optimal impulsive optimization problem: 
Problem 3. Given $N \geq 3, \nu_{1}<\ldots<\nu_{N} \in \mathbb{R}_{>0}, D\left(\nu_{1}\right) \in \mathbb{R}^{6}$, $e \in \mathbb{R}_{[0,1]}, a, \mu \in \mathbb{R}_{>0}$, find $\Delta V^{*} \in \mathbb{R}^{3 N}$ such that:

$$
\begin{array}{cl}
\Delta V^{*}=\underset{\Delta V}{\operatorname{argmin}} & J(\Delta V) \\
\text { s.t. } & \left\{\begin{array}{l}
D\left(\nu_{1}\right)=D_{1} \\
D^{+}\left(\nu_{N}\right)=\Phi_{D}\left(\nu_{N}, \nu_{1}\right) D\left(\nu_{1}\right)+M\left(\nu_{1}, \ldots, \nu_{N}\right) \Delta V \\
d_{0}^{+}\left(\nu_{N}\right)=0 \\
g_{w}\left(D^{+}\left(\nu_{N}\right)\right) \leq 0, w \in\{\underline{x}, \bar{x}, \underline{y}, \bar{y}, \underline{z}, \bar{z}\}
\end{array}\right.
\end{array}
$$

This problem is characterized by convex but non-differentiable functions in its criterion and in the description of its feasible set. It can be converted into an unconstrained optimization problem via penalty methods and the resulting problem can be solved by sub-gradient methods (see [36] for details).

\subsection{Systematical satisfaction of the periodicity constraint}

Hereafter, we show that the periodicity equality constraint $d_{0}^{+}\left(\nu_{N}\right)=0$ can be systematically satisfied by considering only sequences of impulsive velocity corrections that produce periodic relative trajectories. Proceeding this way, the previous mentioned equality constraint need not be taken into account by the numerical solvers while solving Problems $\mathcal{P}, \mathcal{P}$.SDP or $\mathcal{P}$.ENV.

The idea consists in building a basis for the affine subspace to which the vectors of impulsive velocity corrections such that $d_{0}^{+}\left(\nu_{N}\right)=0$ belong. In order to do so, let us recall the first line of (15):

$$
d_{0}^{+}\left(\nu_{N}\right)=d_{0}\left(\nu_{1}\right)+\sum_{i=1}^{N} \frac{1}{k^{2}\left(e^{2}-1\right)}\left[\rho_{\nu_{i}}-e s_{\nu_{i}}\right] \Delta V_{x z}\left(\nu_{i}\right) .
$$

By defining the row vector

$$
M_{0}\left(\nu_{1}, \ldots, \nu_{N}\right):=\frac{1}{k^{2}\left(e^{2}-1\right)}\left[\begin{array}{lllll}
\rho_{\nu_{1}}, & -e s_{\nu_{1}}, & \ldots & \rho_{\nu_{N}}, & -e s_{\nu_{N}}
\end{array}\right],
$$

we can rewrite the equation (31) as:

$$
d_{0}^{+}\left(\nu_{N}\right)=d_{0}\left(\nu_{1}\right)+M_{0}\left(\nu_{1}, \ldots, \nu_{N}\right) \Delta V_{x z} .
$$

Then we express $\Delta V_{x z}$ as:

$$
\Delta V_{x z}(\lambda)=\underbrace{\left[\begin{array}{ccc}
\mid & & \mid \\
v_{1} & \ldots & v_{2 N-1} \\
\mid & & \mid
\end{array}\right]}_{M_{0}^{\perp}\left(\nu_{1}, \ldots, \nu_{N}\right)} \lambda+\Delta V_{0},
$$


where $M_{0}^{\perp}\left(\nu_{1}, \ldots, \nu_{N}\right) \in \mathbb{R}^{2 N \times 2 N-1}, \lambda \in \mathbb{R}^{2 N-1}$ and $\Delta V_{0} \in \mathbb{R}^{2 N}$. The vector $\Delta V_{0} \in \mathbb{R}^{2 N}$ is an arbitrary sequence of in-plane impulses producing a periodic relative trajectory and $M_{0}^{\perp}\left(\nu_{1}, \ldots, \nu_{N}\right) \lambda$ represents a linear combination of the $v_{i}$ vectors belonging to the kernel of the row vector $M_{0}\left(\nu_{1}, \ldots, \nu_{N}\right)$, given by:

$$
v_{i}:=[\underbrace{0, \ldots 0}_{(i-1) \text { zeros }}, a_{i+1}, \quad-a_{i}, \underbrace{0, \ldots 0}_{(2 N-1-i) \text { zeros }}]^{T},
$$

where $a_{i}$ is the i-th entry of $M_{0}\left(\nu_{1}, \ldots, \nu_{N}\right)$.

The idea is a generalization of the developments presented in [38, Section IV.A], where a control law that forces the relative motion between spacecraft to evolve along periodic orbits is described. In this work, we opt to not to constrain the relative motion during the application of the intermediary velocity corrections of a sequence of impulses, only at the final instant. This choice provides more freedom for the research of solutions during the resolution of the optimization problem $\mathcal{P}$.

\section{Model Predictive Control Strategy}

In this section we present a model predictive control algorithm which computes a sequence of saturation constrained impulses, such that the state $D$ converges to a point belonging to a given non-empty admissible set $S_{D}$.

The proposed strategy relies on the following properties, which hold in absence of saturation constraints:

(i) One impulse is sufficient to reach the space of periodic orbits i.e., $d_{0}=0$. Moreover, since periodic trajectories produce no drift (cf. Remark 2.2), the state vector $D$ does not evolve if $d_{0}=0$. These points correspond to the equilibrium points in the $D$ state space.

(ii) Problem $\mathcal{P}$ is always feasible i.e., three impulses separated by a true anomaly interval $\tau_{I} \neq k \pi, k \in$ $\mathbb{N}$ are sufficient for reaching $S_{D}$.

One key contribution is to effectively combine these properties in order to also account for saturation constraints and formally prove the stability and invariance of the proposed method.

For that, an important intermediary result proved in Prop. 3.4 is that one can obtain a sequence of possibly saturated impulses which bring the D state closer to the admissible set, once periodicity is achieved. In sum, the key steps are the following (they are formally summarized in Algorithm 1):

1. Solve Problem $\mathcal{P}$ (in the absence of saturation constraints). If the obtained solution complies with the saturation constraints, the convergence is directly obtained. Otherwise, proceed to next step.

2. Check whether the system is already on a periodic orbit $\left(d_{0}=0\right)$. If so, apply the sequence of impulses obtained at Step 1, with a scaling of the solution. Otherwise, steer the system towards the set of periodic orbits, using (i), with a possible scaling of the solution in case of saturation. 
3. Recursively call Steps 1 and 2 until the state $D$ reaches $S_{D}$.

This is illustrated in Fig. 3: the admissible set $S_{D}$ is unreachable at Step 1 due to saturation. In Step 2 , the algorithm proceeds by driving $d_{0}$ towards 0 (red triangles). Once $d_{0}=0$ (green triangles), one applies the impulses obtained at Step 1 (with possible scaling). The iteration of Step 1-Step 2 stops when the admissible set is reached.

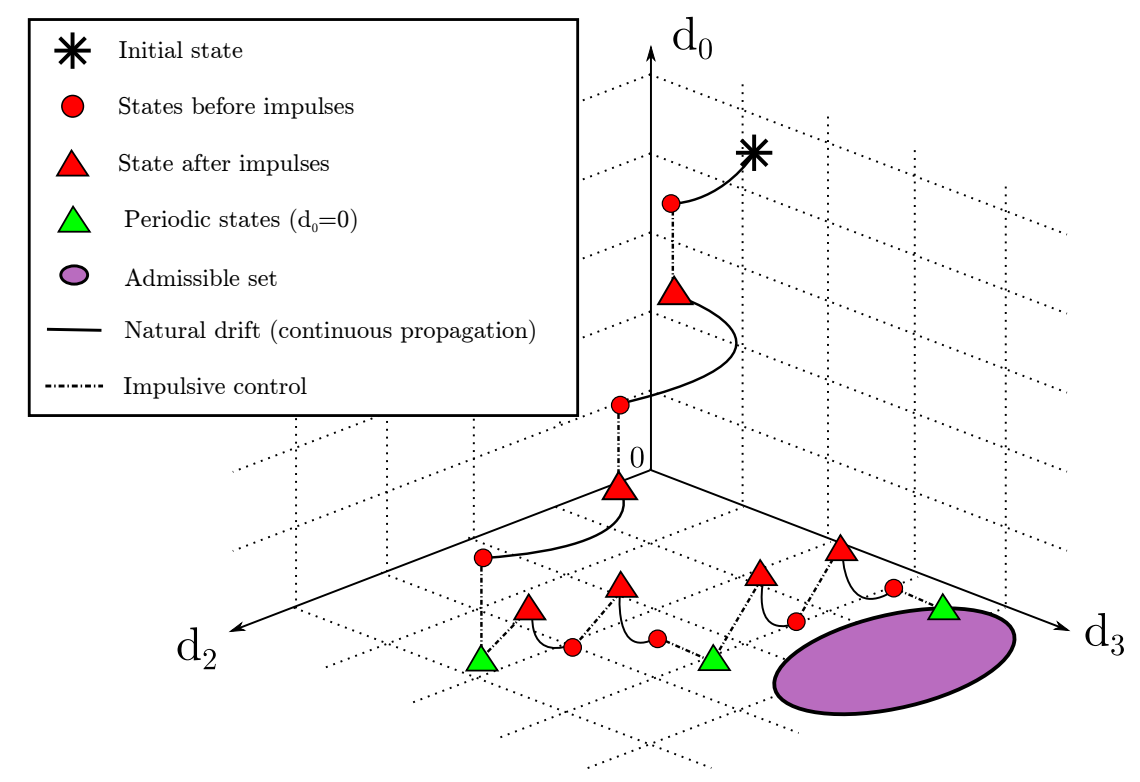

Figure 3: Generation of an admissible periodic trajectory.

This strategy is described in Algorithm 1. It takes as input a relative state in the LVLH framework

Algorithm 1: Model predictive control strategy

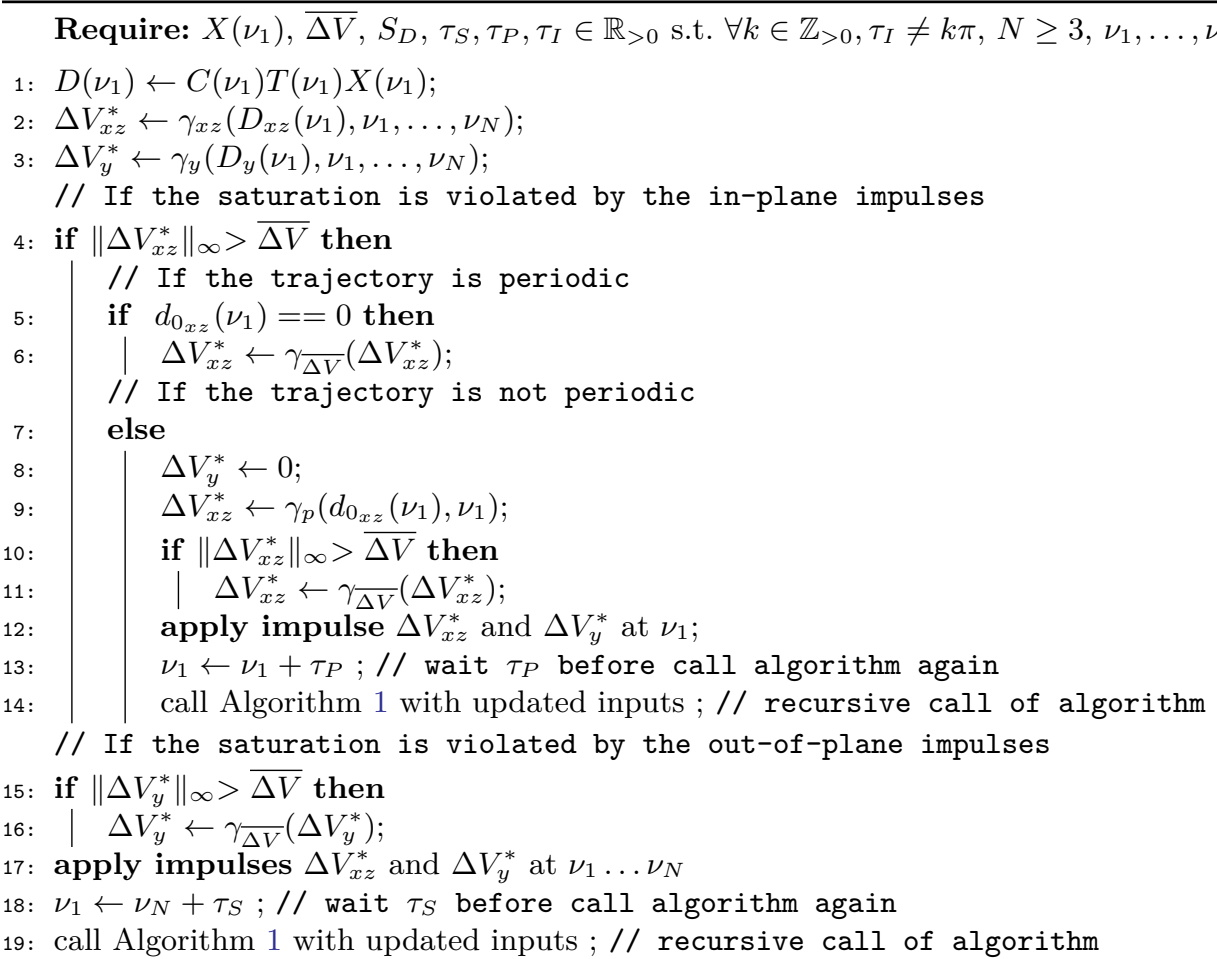


$X\left(\nu_{1}\right)$, a saturation threshold $\overline{\Delta V}$, a non-empty admissible set $S_{D}$ described by $\underline{x}, \bar{x}, \underline{y}, \bar{y}, \underline{z}, \bar{z}$, a number of impulses $N$, an initial true anomaly instant $\nu_{1}$, and the true anomaly intervals $\tau_{P}, \tau_{I}$, and $\tau_{S}$.

While the trajectory is not periodic, the state $D$ evolves within time. In order to generate a periodic trajectory and eliminate this 'drift' effect, some impulses computed by the function $\gamma_{p}$ (defined in Prop. 3.1) are initially applied with the only goal of reducing the absolute value of $d_{0}$. The interval between these impulses is the above mentioned $\tau_{P}$, which should be as small as possible in order to provide a fast convergence to a periodic relative trajectory.

Once periodicity is reached, at each call of the control algorithm, the functions $\gamma_{y}$ and $\gamma_{x z}$ (defined in Prop. 3.2) compute a sequence of a $N$ impulses separated by a true anomaly interval $\tau_{I}$. This interval should not be too small in order to avoid the columns of the matrix $M\left(\nu_{1}, \ldots, \nu_{N}\right)$ of being numerically close to each other, which could possibly demand higher control effort in order to generate admissible relative trajectories (this is confirmed by the simulations presented in the Section 4).

While the admissible set is not reached, sequences of $N$ impulses are consecutively computed and applied. A new sequence can be applied right after the last applied impulse or within some true anomaly interval, given by $\tau_{S}$. A study of the impact of the choice of this interval on the fuel-consumption is carried out in Section 4.

Consecutive calls of this algorithm produce a pattern of impulsive velocity corrections similar to the one presented in Fig. 4.

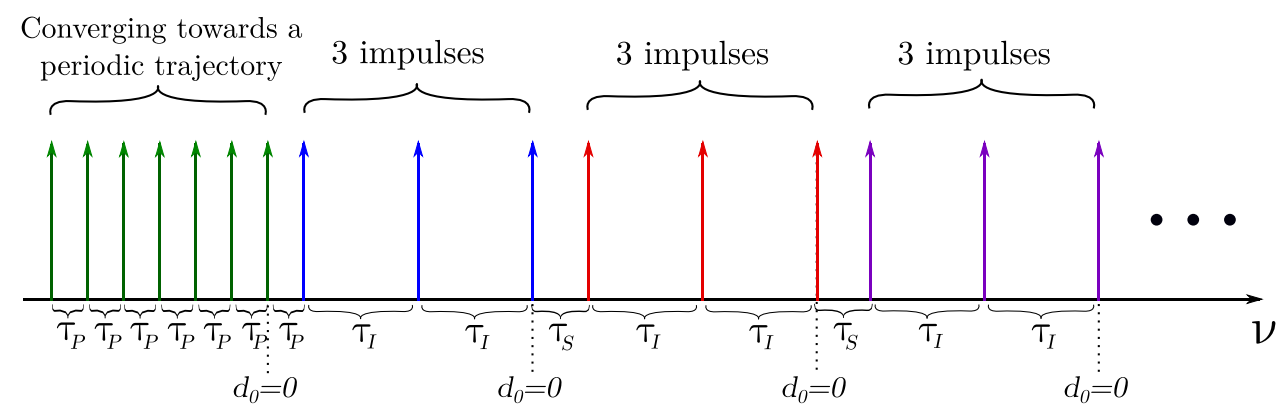

Figure 4: Pattern of impulsive velocity corrections along the true anomaly for a number of impulses $N=3$.

\subsection{Recursive feasibility}

We now prove the recursive feasibility of the optimization problems solved in Algorithm 1 by demonstrating that the functions $\gamma_{p}, \gamma_{y}$ and $\gamma_{x z}$ (defined in the sequel), always return an output for any set of inputs.

Firstly, the function $\gamma_{p}$ computes an $\ell_{1}$-optimal in-plane thrust generating a periodic relative trajectory. 
Proposition 3.1 (Optimal periodic impulse). Consider the function:

$$
\begin{array}{lcll}
\gamma_{p}: & \mathbb{R} \times \mathbb{R} & \rightarrow \mathbb{R}^{2} & \\
d_{0_{x z}}\left(\nu_{1}\right), \nu_{1} & \mapsto \quad \operatorname{argmin}_{\Delta V_{x z}} & \left\|\Delta V_{x z}\right\|_{1} \\
& & \text { s.t. } & d_{0_{x z}}^{+}\left(\nu_{1}\right)=d_{0_{x z}}\left(\nu_{1}\right)+M_{0}\left(\nu_{1}\right) \Delta V_{x z}\left(\nu_{1}\right)=0
\end{array}
$$

Then, for any set of inputs, the function $\gamma_{p}$ is well-defined in the sense that the feasible set of the minimization problem is not empty.

Proof. The line vector $M_{0}\left(\nu_{1}\right)$ has the following expression:

$$
M_{0}\left(\nu_{1}\right)=\left(k^{2}\left(e^{2}-1\right)\right)^{-1}\left[\begin{array}{ll}
1+e \cos \left(\nu_{1}\right) & -e \sin \left(\nu_{1}\right)
\end{array}\right]
$$

and since the term $1+e \cos \left(\nu_{1}\right) \neq 0, \forall \nu_{1}$ (because $0<e<1$ ), it is always possible to set:

$$
\Delta V_{x z}=k^{2}\left(e^{2}-1\right)\left[\begin{array}{ll}
-\frac{d_{0_{x z}}\left(\nu_{1}\right)}{1+e \cos \left(\nu_{1}\right)} & 0
\end{array}\right]^{T}
$$

satisfying the equation $0=d_{0_{x z}}\left(\nu_{1}\right)+M_{0}\left(\nu_{1}\right) \Delta V_{x z}$.

Remark 3.1. Since the minimization problem in (35) contains $\ell_{1}$-norm criteria (which are not strictly convex), infinitely many solutions may exist. In order to enforce uniqueness, we could have taken the solution with minimal $\ell_{2}$-norm (which is strictly convex), but for the sake of brevity, we consider in the sequel that in these special cases only one minimum is arbitrarily chosen.

Secondly, the functions $\gamma_{y}$ and respectively $\gamma_{x z}$ compute an $\ell_{1}$-optimal out-of-plane and respectively in-plane sequence of thrusts which solves Problem $\mathcal{P}$.

Proposition 3.2 (Optimal in- and out-of-plane impulses). Given $N \geq 3, \tau_{I} \in \mathbb{R}_{>0}$ s.t. $\forall k \in \mathbb{Z}_{>0}, \tau_{I} \neq$ $k \pi$, and $\nu_{1}, \ldots, \nu_{N}$ s.t. $\nu_{k+1}=\nu_{k}+\tau_{I}$, let

$$
\begin{aligned}
& \gamma_{y}: \quad \mathbb{R}^{2} \times \mathbb{R} \times \ldots \times \mathbb{R} \rightarrow \mathbb{R}^{N} \\
& D_{y}\left(\nu_{1}\right), \nu_{1}, \ldots, \nu_{N} \mapsto \underset{\Delta V_{y}}{\operatorname{argmin}}\left\|\Delta V_{y}\right\|_{1} \\
& \text { s.t. } \quad D_{y}^{+}\left(\nu_{N}\right)=D_{y}\left(\nu_{1}\right)+M_{y}\left(\nu_{1}, \ldots, \nu_{N}\right) \Delta V_{y} \in S_{D_{y}}
\end{aligned}
$$

and

$$
\begin{aligned}
\gamma_{x z}: \quad \mathbb{R}^{4} \times \mathbb{R} \times \ldots \times \mathbb{R} & \rightarrow \mathbb{R}^{2 N} \\
D_{x z}\left(\nu_{1}\right), \nu_{1}, \ldots, \nu_{N} & \mapsto \underset{\Delta V_{x z}}{\operatorname{argmin}}\left\|\Delta V_{x z}\right\|_{1} \\
& \text { s.t. } D_{x z}^{+}\left(\nu_{N}\right)=D_{x z}\left(\nu_{1}\right)+M_{x z}\left(\nu_{1}, \ldots, \nu_{N}\right) \Delta V_{x z} \in S_{D_{x z}}
\end{aligned}
$$


Then, the functions $\gamma_{y}$ and $\gamma_{x z}$ are well-defined in the sense that the feasible set of (36) and respectively (37) is not empty.

Proof. The detailed proof is given in Appendix A.2. For $\gamma_{y}$, it suffices to note $M_{y}\left(\nu_{1}, \ldots, \nu_{N}\right)$ has rank 2 and that $\forall D_{y}, D_{y}^{*} \in \mathbb{R}^{2}$ the vector $\Delta V_{y}=\left(M_{y}^{T} M_{y}\right)^{-1} M_{y}^{T}\left(D_{y}^{*}-D_{y}\right)$ is well-defined and satisfies $D_{y}^{*}=D_{y}+M_{y}\left(\nu_{1}, \ldots, \nu_{N}\right) \Delta V_{y}$.

For $\gamma_{x z}$, let $D_{x z}, D_{x z}^{*} \in \mathbb{R}^{4}$. We prove that it is always possible to choose $\lambda$ and $\Delta V_{0}$ such that $\Delta V_{x z}(\lambda)=M_{0}^{\perp}\left(\nu_{1}, \ldots, \nu_{N}\right) \lambda+\Delta V_{0}$ (see eq. (33)) satisfies $D_{x z}^{*}=D_{x z}+M_{x z}\left(\nu_{1}, \ldots, \nu_{N}\right) \Delta V_{x z}(\lambda)$. This is based on Prop. 3.1, which allows to set the first entry of $D_{x z}^{*}-D_{x z}$ to any arbitrary value and on the fact that the matrix $M_{x z}\left(\nu_{1}, \ldots, \nu_{N}\right) M_{0}^{\perp}\left(\nu_{1}, \ldots, \nu_{N}\right)$ has rank 3, which allows to set the other 3 entries of $D_{x z}^{*}-D_{x z}$.

Remark 3.2. Similarly, in the minimization problems (36) and (37), one arbitrary minimum is chosen when the problem is not strictly convex.

\subsection{Convergence and invariance}

In this section we prove the stability of the previously described control strategy by demonstrating that the iterative application of the command actions computed in Algorithm 1 produces a sequence of states $\left(D_{k}\right)_{k \in \mathbb{N}}$ that converges to an element of $S_{D}$. We also show that the admissible set is proved to be invariant under the action of the proposed controller, which guarantees that the state remains in the admissible set once the convergence is established. In Algorithm 1, if no saturation occurs, the convergence of the state $D$ to the admissible set $S_{D}$ is trivial. However, for the cases in which the magnitude of the computed impulses goes beyond the saturation threshold, the following ad hoc function is employed to scale-down the sequences of impulses computed either by $\gamma_{p}$ or by $\gamma_{y}$ and respectively $\gamma_{x z}$ in Algorithm 1:

$$
\begin{aligned}
\gamma \overline{\Delta V}: \quad \mathbb{R}^{n} \backslash\{\overrightarrow{0}\} & \rightarrow \mathbb{R}^{n} \\
v & \mapsto \frac{\overline{\Delta V}}{\|v\|_{\infty}} v .
\end{aligned}
$$

Whence, we split the proof of convergence in two parts: first we show that Algorithm 1 produces a periodic relative trajectory; then, once periodicity is obtained, we prove that the algorithm produces a sequence of trajectories that converges to element of $S_{D}$ (in the presence of saturation constraints).

\subsubsection{Convergence}

Let us begin by studying the case in which the sequence of impulses computed at line 2 of Algorithm 1 does not respect the saturation threshold and the initial state $D_{x z}$ is not periodic. In this case, the sequence described in lines 7-14 is executed with the goal of generating a periodic trajectory. In the following proposition, we demonstrate that the sequence $\left(\theta_{k}\right)_{k \in \mathbb{N}}$, representing the evolution of the first 
entry of the state vector $D_{x z}$ (in-plane motion), during recursive calls of Algorithm 1 in the previously described context, converges to zero:

Proposition 3.3 (Convergence to a periodic trajectory). Let be $d_{0_{x z}} \in \mathbb{R}, \nu \in \mathbb{R}, \tau_{P} \in \mathbb{R}_{>0}$. Then, the sequence $\left(\theta_{k}\right)_{k \in \mathbb{N}}$ defined by:

$$
\left(\theta_{k}\right)_{k \in \mathbb{N}}:=\left\{\begin{array}{ll}
\theta_{0}=d_{0_{x z}}, & \\
\theta_{k}=\theta_{k-1}+M_{0}\left(\nu_{k}\right) \Delta V_{x z_{k}}, & \text { if }\left\|\Delta V_{x z_{k}}\right\|_{\infty} \leq \overline{\Delta V} \\
\theta_{k}=\theta_{k-1}+M_{0}\left(\nu_{k}\right) \gamma_{\overline{\Delta V}}\left(\Delta V_{x z_{k}}\right), & \text { if }\left\|\Delta V_{x z_{k}}\right\|_{\infty}>\overline{\Delta V}
\end{array},\right.
$$

where $\Delta V_{x z_{k}}=\gamma_{p}\left(\theta_{k-1}, \nu_{k}\right)$ and $\nu_{k}=\nu+(k-1) \tau_{P}$, converges to 0 .

Proof. Similar to Proof of Prop. 3.5 given in Appendix A.3.

Now we study the convergence of the generated periodic state to the admissible set. Firstly, a preliminary result states that once the periodicity is achieved, a sequence of $N \geq 3$ saturated impulses solution of Problem $\mathcal{P}$ drives the state $D$ closer (and closer) to the admissible set.

Proposition 3.4. Given $N \geq 3, \tau_{I} \in \mathbb{R}_{>0}$ s.t. $\forall k \in \mathbb{Z}_{>0}, \tau_{I} \neq k \pi$, and $\nu_{1}, \ldots, \nu_{N}$ s.t. $\nu_{k+1}=\nu_{k}+\tau_{I}$, and $D\left(\nu_{1}\right)$ a vector representing an arbitrary periodic relative trajectory, let $\Delta V^{*}$ be a solution of Problem $\mathcal{P}$ producing a final state $D^{*}\left(\nu_{N}\right) \in S_{D}$. Let $\overline{\Delta V}$ be a saturation threshold and $D^{\circ}\left(\nu_{N}\right)$ be the final state obtained after a sequence of scaled-down $\gamma_{\overline{\Delta V}}\left(\Delta V^{*}\right)$ impulses. Then,

(a). The state $D^{\circ}\left(\nu_{N}\right)$ represents a periodic orbit;

(b). The state $D^{\circ}\left(\nu_{N}\right)$ is closer to the admissible trajectory $D^{*}\left(\nu_{N}\right)$ than the original $D\left(\nu_{1}\right)$ i.e., $\left\|D^{*}\left(\nu_{N}\right)-D^{\circ}\left(\nu_{N}\right)\right\|_{2}<\left\|D^{*}\left(\nu_{N}\right)-D\left(\nu_{1}\right)\right\|_{2}$.

Proof. Since the states $D$ representing periodic orbits do not freely evolve within time,

$$
D^{*}\left(\nu_{N}\right)=\Phi_{D}\left(\nu_{N}, \nu_{1}\right) D\left(\nu_{1}\right)+M\left(\nu_{1}, \ldots, \nu_{N}\right) \Delta V^{*}=D\left(\nu_{1}\right)+M\left(\nu_{1}, \ldots, \nu_{N}\right) \Delta V^{*},
$$

and similarly, $D^{\circ}\left(\nu_{N}\right)=D\left(\nu_{1}\right)+\eta M\left(\nu_{1}, \ldots, \nu_{N}\right) \Delta V^{*}$, with the scaling factor $\eta=\frac{\overline{\Delta V}}{\left\|\Delta V^{*}\right\|_{\infty}}, 0<\eta \leq 1$.

From equation (33), any sequence of impulses generating a periodic trajectory can be expressed as: $\Delta V=M_{0}^{\perp}\left(\nu_{1}, \ldots, \nu_{N}\right) \lambda+\Delta V_{0}$, where $\Delta V_{0}$ represents the part of the impulses generating a periodic orbit and $M^{\perp}\left(\nu_{1}, \ldots, \nu_{N}\right) \lambda$ represents the part of the impulses that has no influence on $d_{0}$. Since $\Delta V^{*}$ generates a periodic orbit $D^{*}\left(\nu_{N}\right)$ from another periodic orbit $D\left(\nu_{1}\right), \Delta V_{0}^{*}=0$, whence:

$$
D^{*}\left(\nu_{N}\right)=D\left(\nu_{1}\right)+M\left(\nu_{1}, \ldots, \nu_{N}\right) M_{0}^{\perp}\left(\nu_{1}, \ldots, \nu_{N}\right) \lambda^{*}
$$




$$
D^{\circ}\left(\nu_{N}\right)=D\left(\nu_{1}\right)+\eta M\left(\nu_{1}, \ldots, \nu_{N}\right) M_{0}^{\perp}\left(\nu_{1}, \ldots, \nu_{N}\right) \lambda^{*}
$$

This proves (a) by construction and (b) follows from simple computation.

Remark 3.3. Although for general MPC strategies one single control action is applied by iteration, our method employs the iterative application of a sequence of at least 3 impulsive velocity corrections, separated by a true anomaly interval that is not a multiple of $\pi$. This choice is justified by Proposition 3.4 stated above.

In the sequel, it is proven that, the sequence $\left(\phi_{k}\right)_{k \in \mathbb{N}}$, representing the iterative application of the control Algorithm 1 on the state $D$, converges to the admissible set:

Proposition 3.5 (Convergence to the admissible set). Let be $D \in \mathbb{R}^{6}$ such that $d_{0}=0, \nu, \tau_{I}, \tau_{S} \in \mathbb{R}_{>0}$ such that $\forall k \in \mathbb{Z}_{>0}, \tau_{I} \neq k \pi, N \geq 3$ and $S_{D} \neq \emptyset$. Then, the following sequence:

$$
\left(\phi_{k}\right)_{k \in \mathbb{N}}:= \begin{cases}\phi_{0}=D, \\ \phi_{k}=\phi_{k-1}+M\left(\nu_{k}^{(1)}, \ldots, \nu_{k}^{(N)}\right) \Delta V_{k}, & \text { if }\left\|\Delta V_{y_{k}}\right\|_{\infty} \leq \overline{\Delta V} \\ \phi_{k}=\phi_{k-1}+M\left(\nu_{k}^{(1)}, \ldots, \nu_{k}^{(N)}\right) \gamma_{\overline{\Delta V}}\left(\Delta V_{k}\right), & \text { if }\left\|\Delta V_{y_{k}}\right\|_{\infty}>\overline{\Delta V}\end{cases}
$$

where $\nu_{k}^{(i)}=\nu+(i-1) \tau_{I}+(k-1) \tau_{S}$ and $\Delta V_{k}$ is the concatenation of $\Delta V_{x z_{k}}=\gamma_{x z}\left(\phi_{k-1}, \nu_{k}^{(1)}, \ldots, \nu_{k}^{(N)}\right)$ $\Delta V_{y_{k}}=\gamma_{y}\left(\phi_{k-1}, \nu_{k}^{(1)}, \ldots, \nu_{k}^{(N)}\right)$, converges to an element of $S_{D}$.

Proof. The detailed proof is given in Appendix A.3. The main idea is to show that the distance between $\phi_{k}$ and $S_{D}$ converges to zero. In order to do so, two cases are studied: in the first case, we suppose that, for a certain $k^{*}$, the computed sequence of impulses satisfies the saturation constraint, generating an admissible periodic orbit right after its application; in the second case, we suppose that all the sequence of impulses must be scaled-down to comply with the saturation constraint. In this case, we show that there exists a constant $0<\alpha<1$ such that $\operatorname{dist}_{S_{D_{y}}}\left(\phi_{k}\right)<\alpha \underset{S_{D}}{\operatorname{dist}}\left(\phi_{k-1}\right)$ and, consequently, $\underset{S_{D}}{\operatorname{dist}}\left(\phi_{k}\right) \underset{k \rightarrow \infty}{\rightarrow} 0$.

\subsubsection{Invariance}

So far we established the convergence of the state $D$ to an element of the admissible set. In this section we demonstrate that, since the set $S_{D}$ is naturally invariant as a subset of the invariant set of states representing periodic orbits, it can also be proved to be an invariant set for the evolution of the state $D$ under the actions of the proposed control law. Conversely, once an admissible trajectory is obtained, it is preserved by the model predictive control algorithm.

Proposition 3.6 (Invariance). The set $S_{D}$ is invariant under the action of the instructions defined in Algorithm 1. 
Proof. This is evident: since $D \in S_{D} \Rightarrow d_{0}=0$ (periodicity), the function $\gamma_{p}$ is never called; moreover, the functions $\gamma_{y}$ and $\gamma_{x z}$ compute the fuel-optimal sequence of impulses that generates a trajectory respecting the out-of-plane and the in-plane space constraints respectively. However, since $D \in S_{D} \Rightarrow \underline{x} \leq x(\nu) \leq \bar{x}, \underline{y} \leq y(\nu) \leq \bar{y}, \underline{z} \leq z(\nu) \leq \bar{z}, \forall \nu$, these functions will return a null sequence of impulses.

\section{Simulations and Results}

Hereafter we present the simulations and results obtained by employing the proposed MPC algorithm to control the relative motion between spacecraft during the rendezvous hovering phases. The simulations performed in this section are divided in two parts, with different goals: the first part is dedicated to the evaluation of general aspects of the proposed MPC algorithm, such as robustness, convergence time, fuel-consumption and the on-board running time of the algorithm during the resolution of optimization problems; in the second part, we aim to study the impact of the eccentricity, number of impulses, initial true anomaly and the three true anomaly intervals $\tau_{P}, \tau_{I}, \tau_{S}$ on the total fuel-consumption.

\subsection{Hardware-in-the-loop simulations}

The tests are performed in a hardware-in-the-loop environment: each call of the MPC algorithm is executed on a board dedicated to space application; the computed control actions are sent via user datagram protocol (UDP) to a computer running a Matlab/Simulink model that simulates the relative dynamics between spacecraft (see Fig. 5).

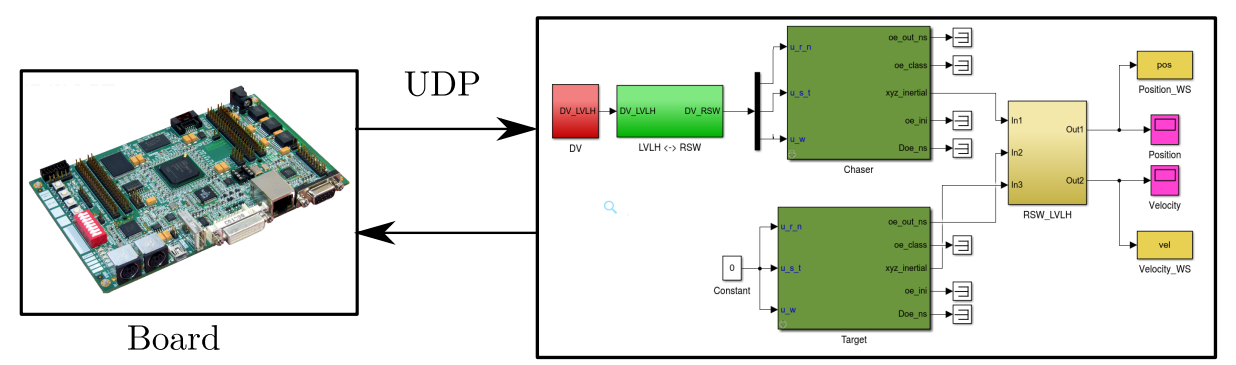

Matlab Simulator

Figure 5: Hardware-in-the-loop environment: network connection scheme between board and simulator.

\subsubsection{Software}

Both the SDP and the envelope (hereafter we use the abbreviation ENV) approaches are adopted to model the fuel-optimal control problem. The SDP problems are solved via the CSDP solver [45], using the standard options and parameters. The envelope problems are solved by a combination of penalty method with iterative optimization algorithms based on sub-gradients: the constraints are weighted by a 
coefficient equivalent to $10^{8}$ and added to the objective function and the resulting unconstrained problem is solved by performing (at most) 50 iterations of the BFGS method ${ }^{2}$ presented by Lewis and Overton [46, Algorithm 2.1], followed by 500 iterations of the sub-gradient method presented by Shor et al. [47, Theorem 2.2] (see [36] for details). All embedded programs are coded in C.

\subsubsection{Hardware}

The board is an AEROFLEX GAISLER GR-XC6S that contains a synthesized LEON3 microprocessor [48] and supports a IEEE-754 compliant floating-point unit with single and double precision (32 and 64-bit floats). It has a 128 Mbyte DDR2 RAM, a 8 Mbyte PROM and a 8 Mbyte SPI PROM memories and runs a Linux 2.6 environment that simulates the performance of devices usually employed in space applications [49]. The embedded libraries occupy 12 Mbyte and the $\mathrm{C}$ binary executables have 44 Kbyte (ENV) and 148 Kbyte (SDP).

\subsubsection{Simulating the relative dynamics}

Two types of simulators are used: a linear simulator computing the evolution of the relative motion via the propagation of the state $D$ presented in equation 18; and a nonlinear simulator [41] based on the Gauss planetary equations for the relative motion $[50,51]$ that takes into account the effects of disturbances, such as the atmospheric drag, Earth's oblateness, uncertainties on the measurement of the relative state (we consider a white noise on position and velocity characterized by the following standard deviation: $d_{p}=10^{-2} \mathrm{~m}, d_{v}=10^{-5} \mathrm{~m} / \mathrm{s}$ ), execution errors on the orientation and magnitude of applied impulsive velocity corrections (we consider a mismatch of $\pm 1^{\circ}$ in orientation and $\pm 1 \%$ in magnitude). The linear simulations are performed in order to verify the theoretical results about the stability of the proposed algorithm. The nonlinear simulations assess the robustness of the proposed algorithm under disturbances and nonlinearities that are not taken into account by the linear model.

\subsubsection{Scenarios}

In order to compare the obtained results to those presented in [38], the same scenarios (based on the PRISMA mission [52]) are studied: Earth's gravitational constant: $\mu=3.986004418 .10^{14} \mathrm{~m}^{3} \mathrm{~s}^{-2}$; leader's orbital parameters: $e=0.004, a=7011 \mathrm{~km}, i=98^{\circ}, \Omega=0^{\circ}, \omega=0^{\circ}$; leader's initial true anomaly $\nu_{0}=0^{\circ}$; number of impulses adopted is $N=3$; true anomaly interval between impulses $\tau_{I}=120^{\circ}$; true anomaly interval between sequence of impulses $\tau_{S}=120^{\circ}$; true anomaly interval to achieve periodicity $\tau_{P}=3,6^{\circ}$; space constraints: $\underline{x}=50 \mathrm{~m}, \bar{x}=150 \mathrm{~m}, \underline{y}=-25 \mathrm{~m}, \bar{y}=25 \mathrm{~m}, \underline{z}=-25 \mathrm{~m}, \bar{z}=25 \mathrm{~m}$;

\footnotetext{
${ }^{2} \mathrm{~A}$ version of the algorithm HANSO v2.2 translated to C is used (http://www.cs.nyu.edu/overton/software/hanso/).
} 
thrusters saturation threshold: $0.5 \mathrm{~m} / \mathrm{s}$; duration of simulation: 10 orbital periods; initial relative state:

$$
\begin{aligned}
& X_{01}=[\quad 400, \quad 300, \quad-40, \quad 0, \quad 0, \quad 0]^{T} \\
& X_{02}=\left[\begin{array}{llllll}
-800, & 600, & 200, & 0, & 0, & 0
\end{array}\right]^{T} \\
& X_{03}=\left[\begin{array}{llllll}
-1500, & 1300, & 150, & 0, & 0, & 0
\end{array}\right]^{T}, \\
& X_{04}=[5000,1300, \quad 500, \quad 0, \quad 0, \quad 0]^{T}
\end{aligned}
$$

where the first three components of each vector represent the relative LVLH positions (in meters) and the last three, the relative LVLH velocities (in meters per second);

\subsection{Results analysis}

\subsubsection{Convergence definition}

To evaluate the convergence, we extend the use the mismatch ratio $\eta$ presented in [38]. The mismatch ratio is given by:

$$
\eta(\nu)=\frac{\operatorname{dist}_{D} D(\nu)}{\operatorname{dist}_{D} D\left(\nu_{0}\right)}=\frac{\operatorname{dist} C(\nu) T(\nu) X(\nu)}{\operatorname{Sist}_{D} C\left(\nu_{0}\right) T\left(\nu_{0}\right) X\left(\nu_{0}\right)},
$$

which is the ratio between the distance to the admissible set of the current and initial state. For a given $\delta \in[0,1]$ the convergence time $T_{c}$ is defined as:

$$
T_{c}(\delta) \in \mathbb{R}_{>0} \text { s.t. } \forall \nu \geq T_{c}, \eta(\nu) \leq \delta
$$

In the results presented hereafter, $\delta$ is set to $5 \%$ and the convergence time is normalized by the orbital period of the leader spacecraft, providing an idea of the number of orbits needed to achieve convergence.

\subsubsection{Consumption, convergence time and running time}

Convergence $(\delta<5 \%)$ and hovering are obtained for all performed simulations. Table 1, present the obtained fuel-consumption $J$. From this point of view, the SDP-based controller is the most performing with respect to the ENV-based controller. This is due to the fact that the limited number of iterations of the BFGS and sub-gradient algorithms generate suboptimal solutions of Problem $\mathcal{P}$.ENV, while the SDP approach always returns the optimal solution of Problem P.SDP. Nevertheless, both approaches engender fuel-consumptions that are approximatively half of the lower values produced by any of the three control laws proposed in [38].

Convergence times $T_{c}$ for each simulations are reported in Table 2. The non linear simulation environment has little impact on convergence performances except for the initial condition $X_{04}$. Comparing with the hybrid controller developed in [38], the proposed approaches are not generally the best. For instance, for $X_{01}$ the control law B in [38] generates a convergence time equal to 0.34 orbits, while the 
proposed SDP and ENV approaches take twice as much time to converge. This indicates that the strategy that we propose gives more emphasis to reducing the consumption than producing short convergence times. Besides, when the initial condition recede from the hovering zone, the MPC controller abilities to account for input constraints permit to ensure the convergence and limit the convergence time. On the other hand, the behavior of hybrid controllers is degraded in terms of convergence and consumption due to the presence of the saturation (one of the examples of application of the hybrid controller even diverges).

Table 1: Consumption $J(\mathrm{~m} / \mathrm{s})$

\begin{tabular}{ccccc}
\hline \hline Initial & \multicolumn{2}{c}{ SDP } & \multicolumn{2}{c}{ ENV } \\
condition & LIN & NLIN & LIN & NLIN \\
\hline$X_{01}$ & 0.45 & 0.47 & 0.53 & 0.58 \\
$X_{02}$ & 1.20 & 1.25 & 1.31 & 1.32 \\
$X_{03}$ & 2.21 & 2.26 & 2.31 & 2.38 \\
$X_{04}$ & 4.75 & 4.71 & 5.41 & 6.69 \\
\hline \hline
\end{tabular}

Table 2: Convergence time $T_{c}$ (number of orbits)

\begin{tabular}{ccccc}
\hline \hline Initial & \multicolumn{2}{c}{ SDP } & \multicolumn{2}{c}{ ENV } \\
condition & LIN & NLIN & LIN & NLIN \\
\hline$X_{01}$ & 0.66 & 0.66 & 0.64 & 0.69 \\
$X_{02}$ & 0.66 & 0.66 & 0.64 & 0.69 \\
$X_{03}$ & 1.65 & 1.65 & 1.65 & 1.65 \\
$X_{04}$ & 2.69 & 1.70 & 1.67 & 2.66 \\
\hline \hline
\end{tabular}

Table 3 and 4 permit to compare the numerical performance both SDP and ENV based controllers. For both approaches, the average time to compute a sequence of $N=3$ impulses is lower than 3.0 seconds and the maximal running time is never longer than 4.0 seconds (this time is negligible when compared to the orbital period $T=2 \pi \sqrt{a^{3} / \mu} \approx 5842$ seconds). Moreover, the amount of memory allocated by the execution of the binaries are 5056 Kbyte for the SDP approach and 5584 Kbyte for the ENV approach - these are reasonable values compared to the available memory of approximatively 90 Mbyte.

Table 3: Average running time (s)

\begin{tabular}{ccccc}
\hline \hline Initial & \multicolumn{2}{c}{ SDP } & \multicolumn{2}{c}{ ENV } \\
condition & LIN & NLIN & LIN & NLIN \\
\hline$X_{01}$ & 2.82 & 2.94 & 0.30 & 2.71 \\
$X_{02}$ & 2.82 & 2.86 & 0.31 & 2.68 \\
$X_{03}$ & 2.83 & 2.87 & 0.41 & 2.52 \\
$X_{04}$ & 2.70 & 2.89 & 1.54 & 2.81 \\
\hline \hline
\end{tabular}

Table 4: Maximal running time (s)

\begin{tabular}{ccccc}
\hline \hline Initial & \multicolumn{2}{c}{ SDP } & \multicolumn{2}{c}{ ENV } \\
condition & LIN & NLIN & LIN & NLIN \\
\hline$X_{01}$ & 2.93 & 3.31 & 3.05 & 2.91 \\
$X_{02}$ & 2.96 & 3.23 & 3.07 & 3.09 \\
$X_{03}$ & 2.93 & 3.05 & 3.23 & 3.12 \\
$X_{04}$ & 2.79 & 3.54 & 3.29 & 3.37 \\
\hline \hline
\end{tabular}

\subsubsection{Relative trajectories, impulses and distance to the admissible set}

In Figures 6 - 9, we show the resulting 3D relative trajectories (we zoom into the hovering region), the computed and applied impulses for the nonlinear simulations and the evolution of the mismatching ratio $\eta(\nu)$ for the initial conditions $X_{03}$ and $X_{04}$. By observing the relative trajectories obtained for the linear simulations, we notice that the relative movement converges to a periodic trajectory included in the hovering zone and, once this trajectory is reached, it remains unchanged - this fact illustrates the convergence and invariance results demonstrated in Prop. 3.5 - 3.6. The same behavior, however, is not observed for nonlinear simulations: due to the presence of disturbances and uncertainties, the control actions are not able to produce perfect periodic orbits. This is also observed in Fig. 10a where for 
the nonlinear simulation, the mismatch ratio oscillates close to zero, but never reaches it. Moreover, although some impulses are saturated (Fig. 6b, 7b, 8b and 9b), the convergence is achieved for both linear and nonlinear simulations.

In Fig. 10b we show in details the four initial impulses applied in order to reduce the absolute value of $d_{0}$ (these impulses are computed via $\gamma_{p}$ and are separated by true anomaly intervals of $\tau_{P}$, indicated in the figure; for nonlinear simulations, due to the disturbances, the condition $d_{0}=0$ is never reached, being therefore replaced by another condition $\left|d_{0}\right|<$ threshold). In Fig. 11 we show that after each sequence of $N=3$ impulses, the distance to the admissible set decreases (indicated by the dotted lines). Furthermore, during the interval between sequences of impulses (indicated by $\tau_{S}$ and the shaded zones), the mismatch ratio remains constant.

\subsubsection{Impact of parameters on fuel-consumption}

Hereafter we study the effect of some parameters (eccentricity, number of impulses, initial true anomaly and the three true anomaly intervals $\left.\tau_{P}, \tau_{I}, \tau_{S}\right)$ on the total fuel-consumption. We perform linear simulations using the SDP approach for the four initial states $X_{01}-X_{04}$; one single parameter varies at time and the others are kept at the same values employed in the previous simulations. The obtained results are presented in Fig. $13-17$.

Fig. 12 indicates that a small number of impulses should be chosen, since the fuel-consumption increases with the growth of this parameter. The augmentation of the fuel-consumption with the increase of the eccentricity (Fig. 13) or with the reduction of the interval between impulses (Fig. 14) are consistent with results previously presented in the literature [43, Section 6.4]. Different choices of initial true anomaly produce a sinusoidal profile for the fuel-consumption, which implies the existence of a fuel-optimal choice for the initial firing instant $\nu_{0}$ (Fig. 15). In Fig. 16, the consumption increases until it reaches its maximum around $\tau_{p}=2.4^{\circ}$ or $0.042 \mathrm{rad}$, then starts to decrease; in order to minimize consumption and convergence time, this parameter should be set to the smallest value possible, which is defined by the physical limitations of the spacecraft thrusters. The profile of consumption obtained by varying the interval between sequences of impulses does not present a particular shape or behavior and therefore no general conclusion can be obtained from it (Fig. 17). Remark: in Fig. 15 - 17, for each initial state, the fuel-consumptions are normalized between 0 and 1.

\section{Conclusions}

In this article, a new model predictive control strategy is proposed for the impulsive spacecraft rendezvous hovering phases. A theoretical stability proof is provided, demonstrating that, even when the saturation of the thrusters is taken into account, the proposed strategy produces a sequence of control actions 


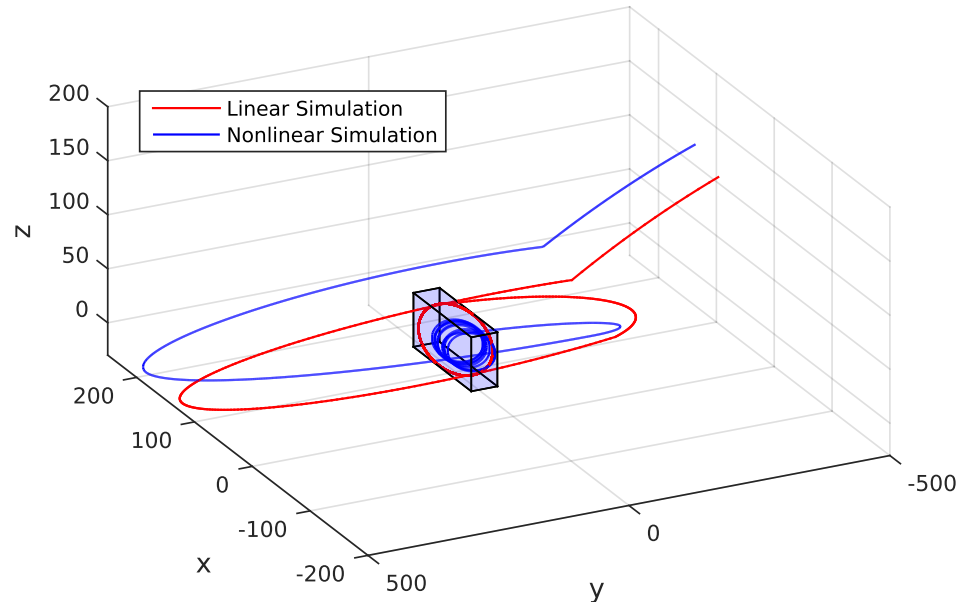

(a) 3D Relative Trajectory.

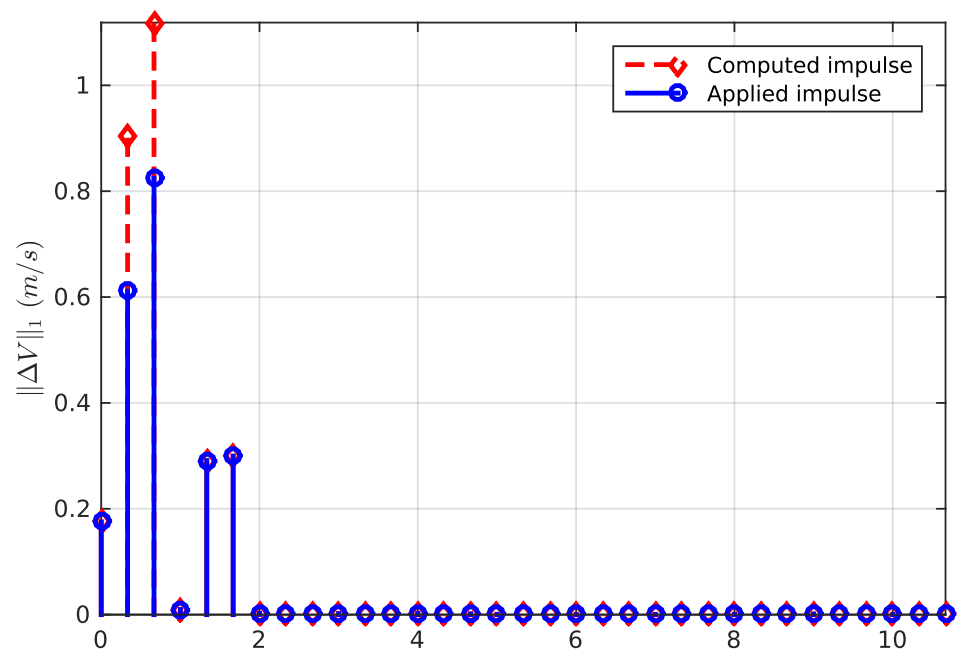

Time in number of orbits

(b) Computed and applied impulses during nonlinear simulation.

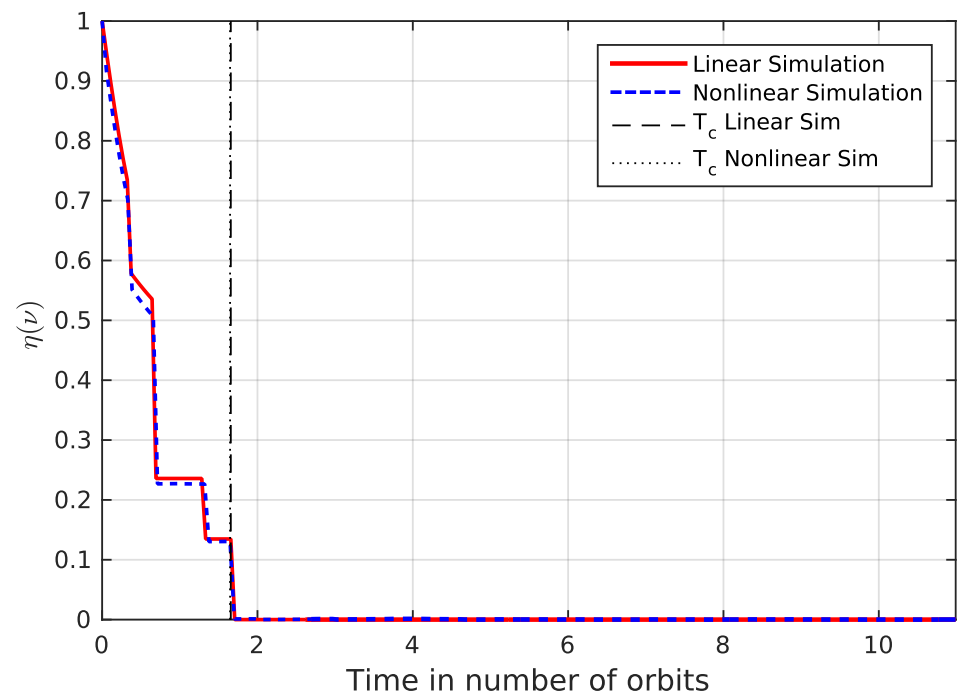

(c) Convergence to admissible set.

Figure 6: Results for trajectory $X_{03}$ (SDP approach). 


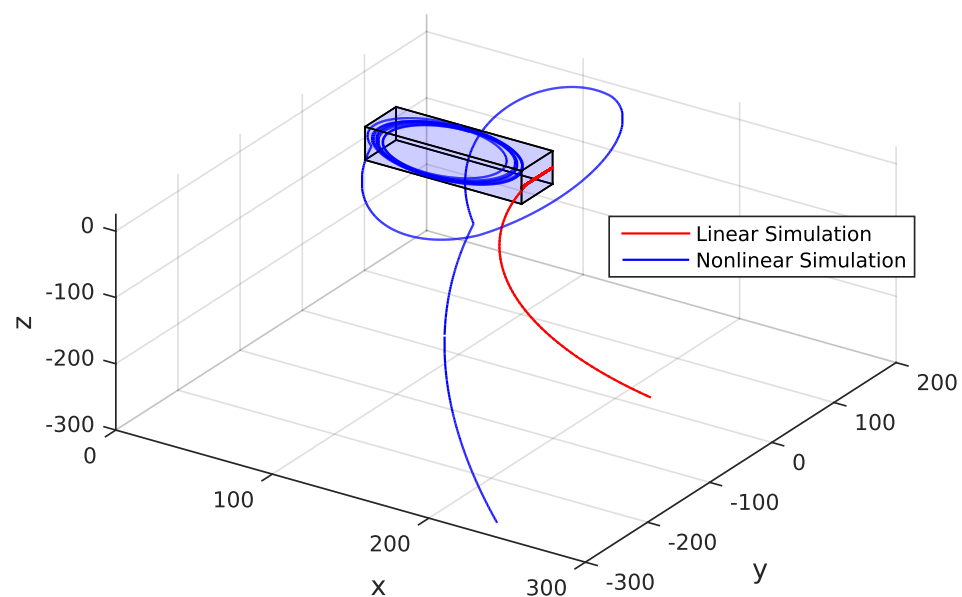

(a) 3D Relative Trajectory.

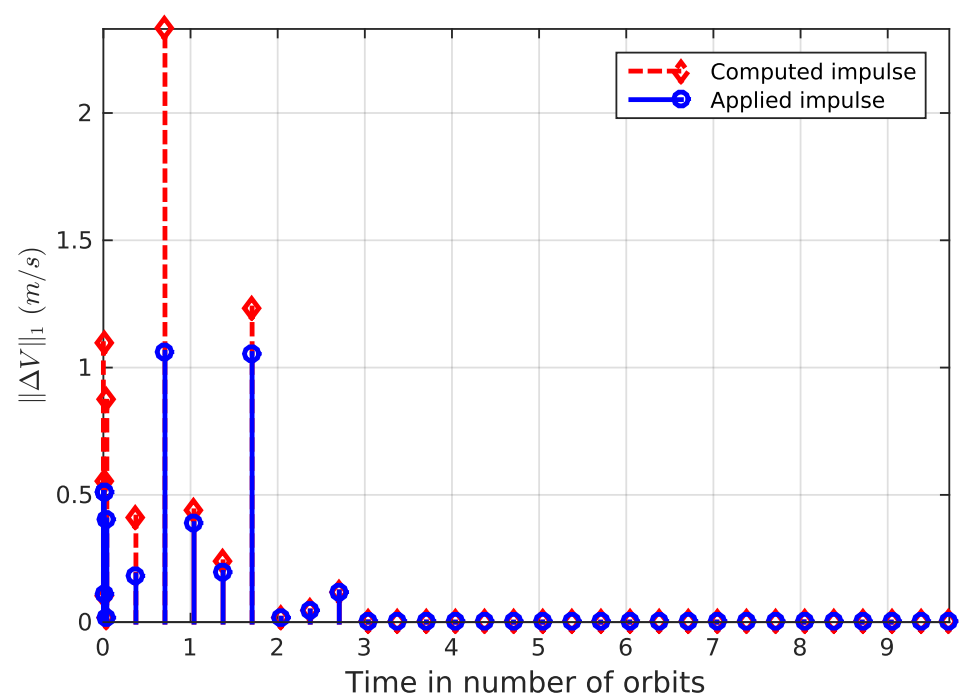

(b) Computed and applied impulses during nonlinear simulation.

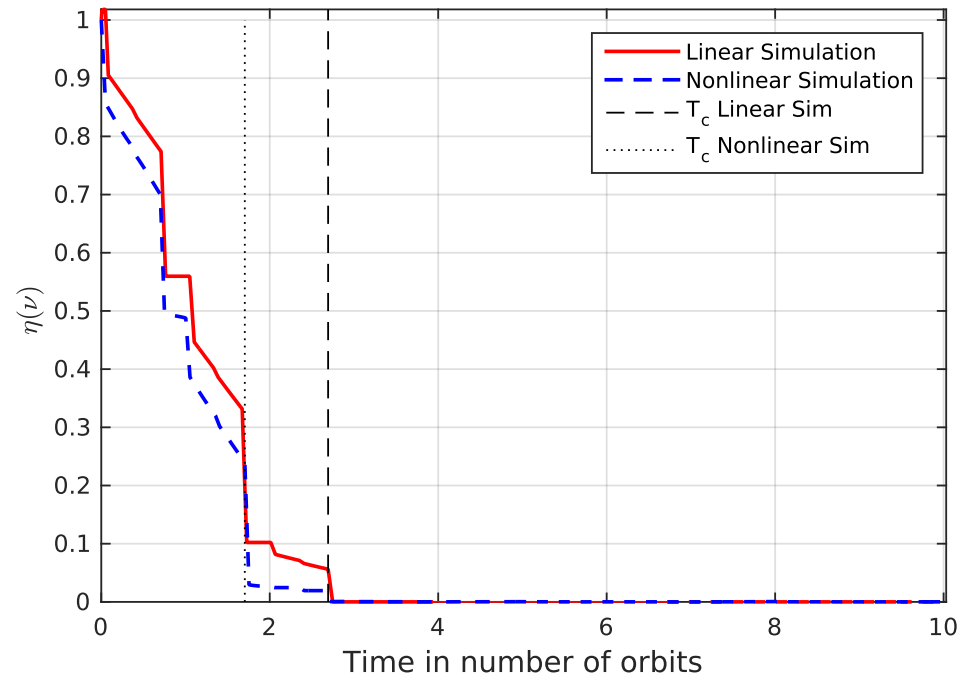

(c) Convergence to admissible set.

Figure 7: Results for trajectory $X_{04}$ (SDP approach). 


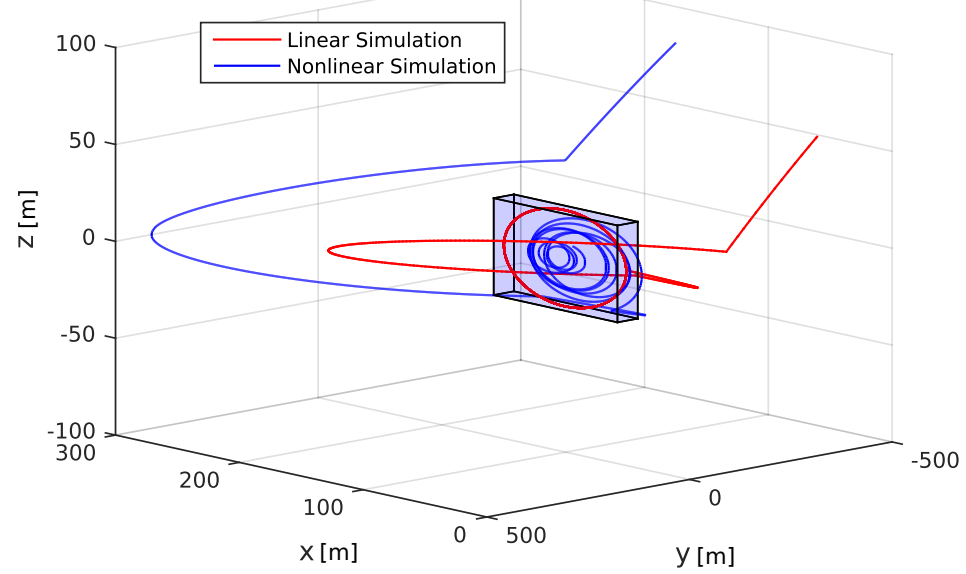

(a) 3D Relative Trajectory.

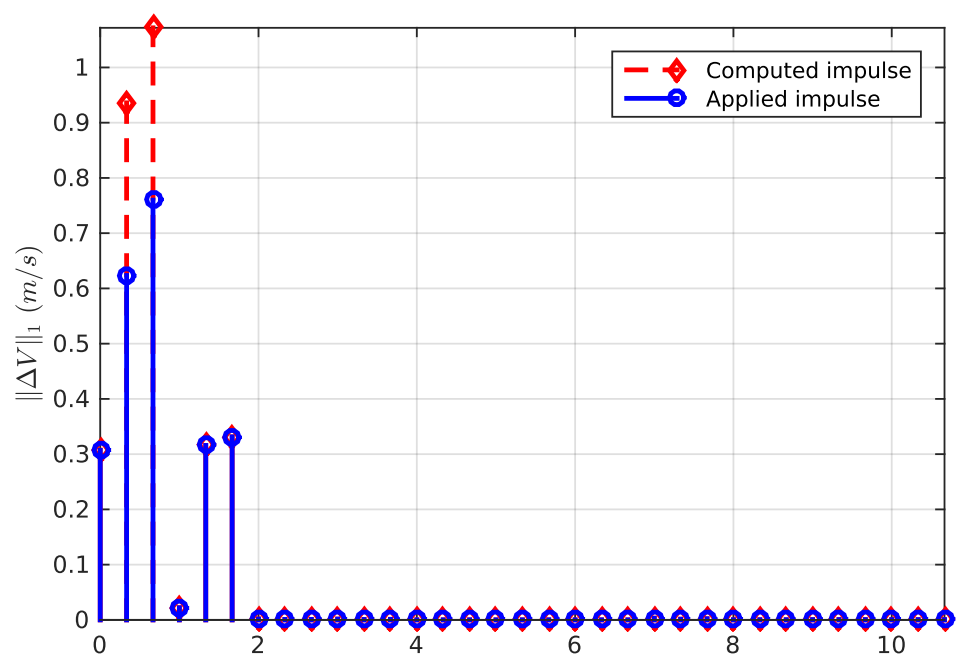

Time in number of orbits

(b) Computed and applied impulses during nonlinear simulation.

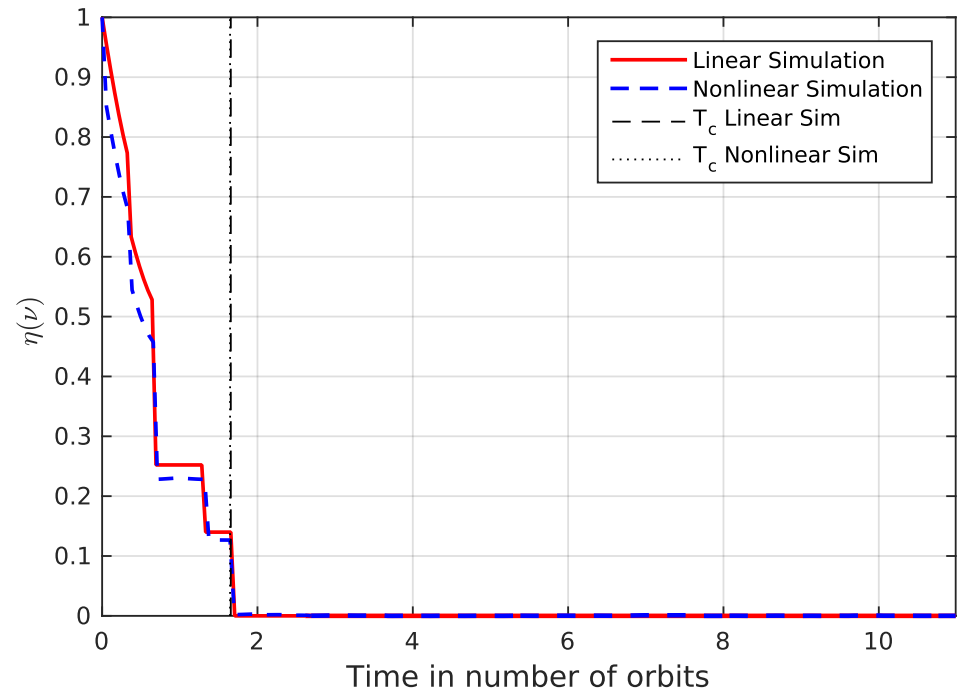

(c) Convergence to admissible set.

Figure 8: Results for trajectory $X_{03}$ (ENV approach). 


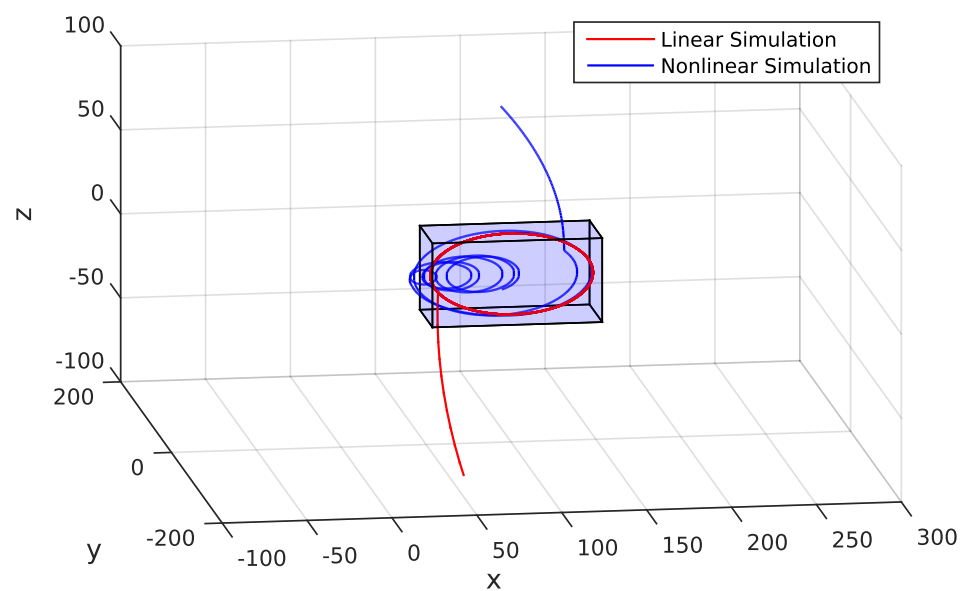

(a) 3D Relative Trajectory.

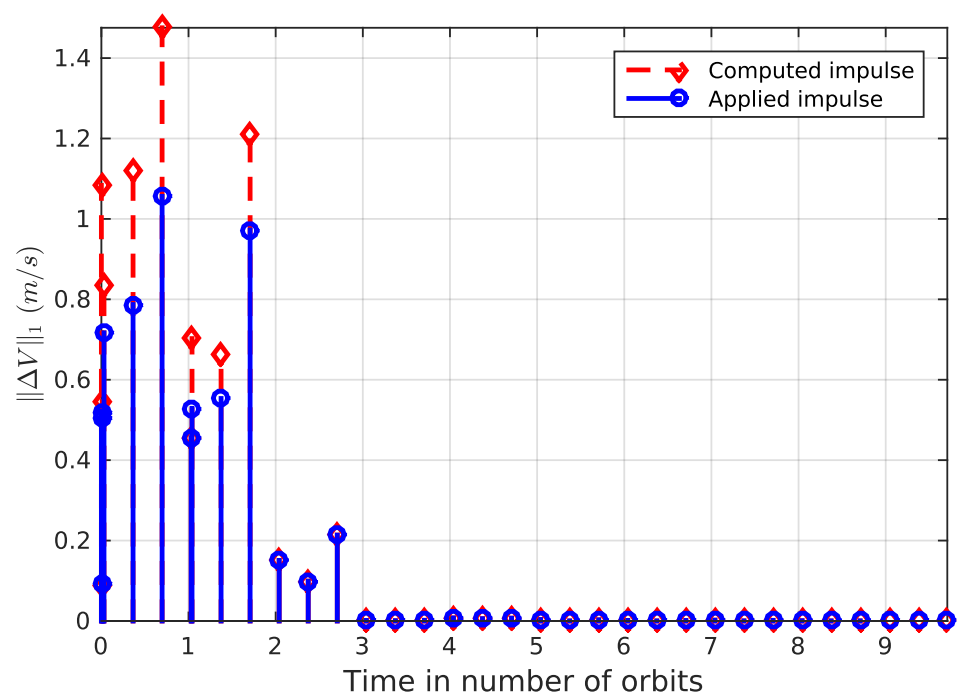

(b) Computed and applied impulses during nonlinear simulation.

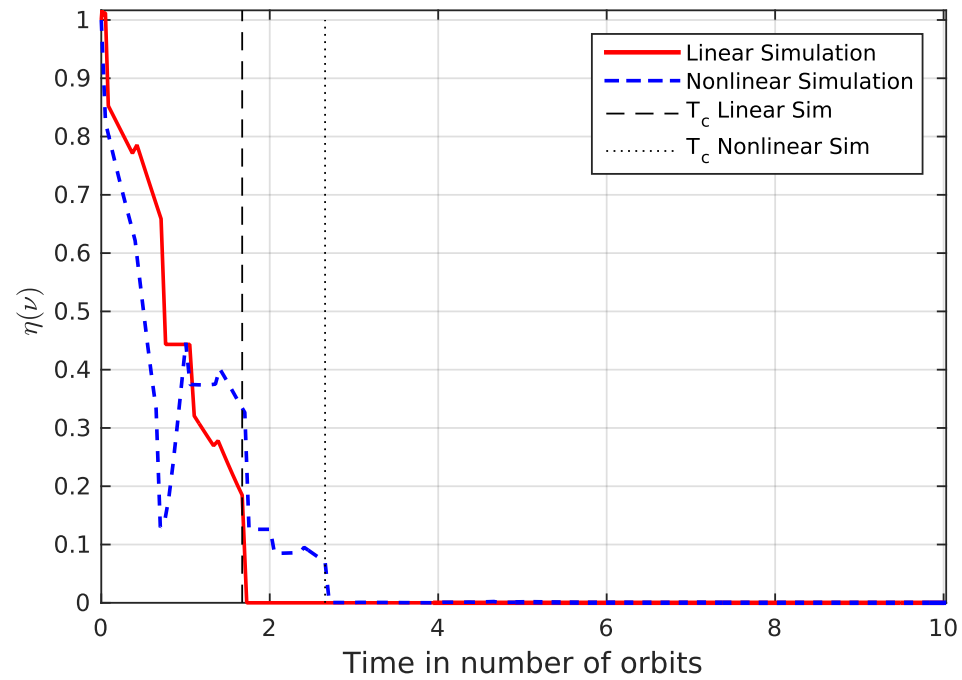

(c) Convergence to admissible set.

Figure 9: Results for trajectory $X_{04}$ (ENV approach). 


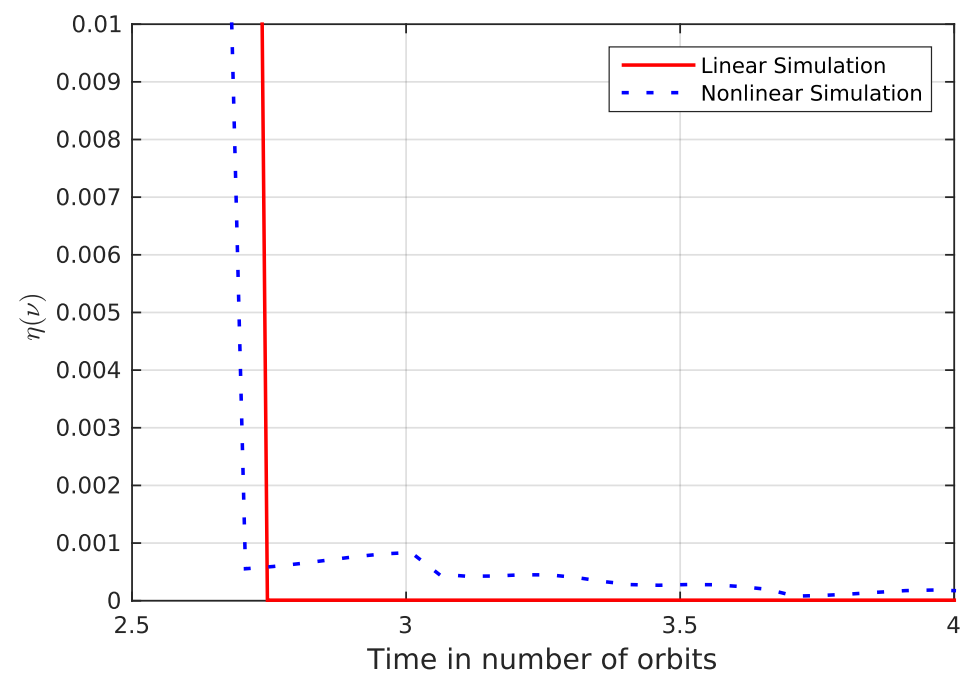

(a) Effect of disturbances in nonlinear simulations.

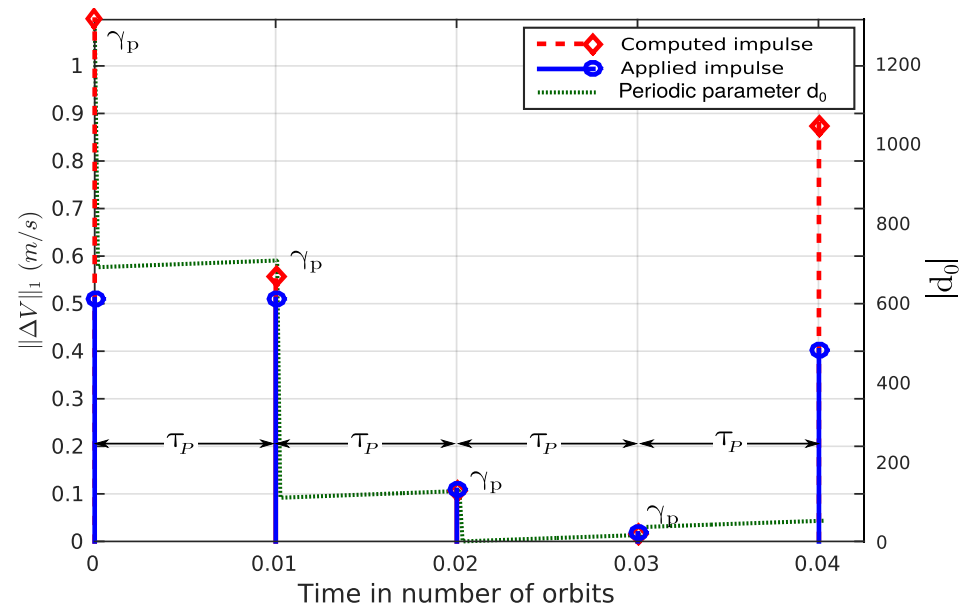

(b) Impulses producing a periodic trajectory.

Figure 10: Details of results obtained for trajectory $X_{04}$ (SDP approach).

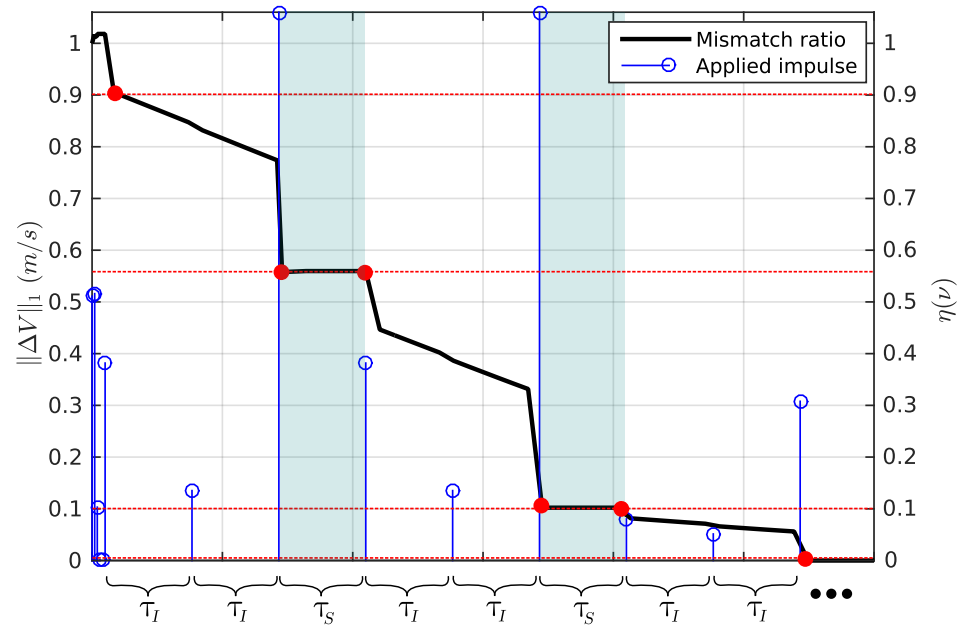

Figure 11: Decrease of the mismatch ratio after each sequence of 3 impulses $\left(X_{04}\right.$, linear simulation, SDP). 


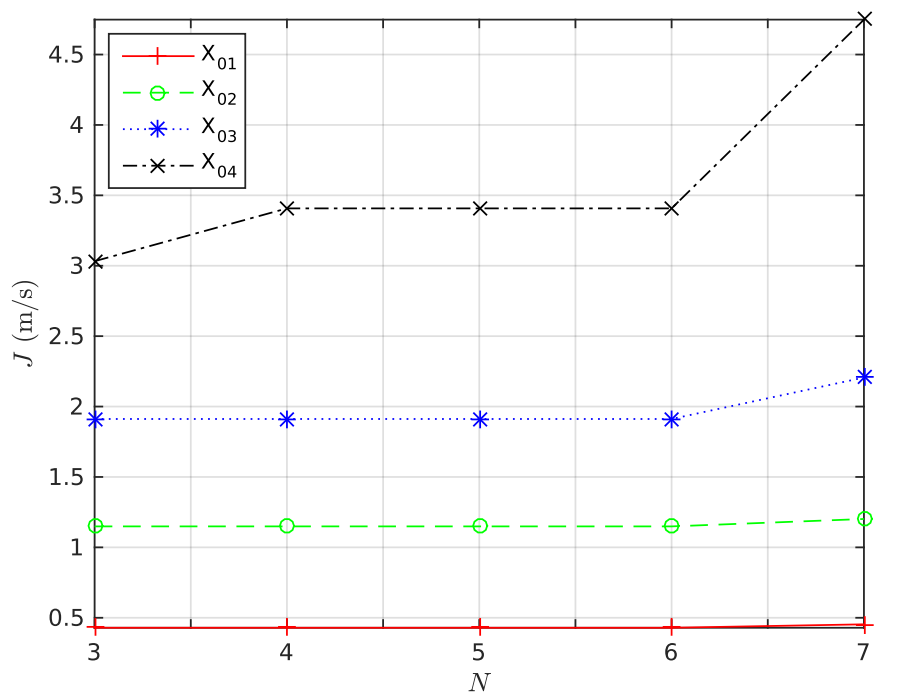

Figure 12: Impact of number of impulses $N$ on the fuel-consumption $J(\Delta V)$.

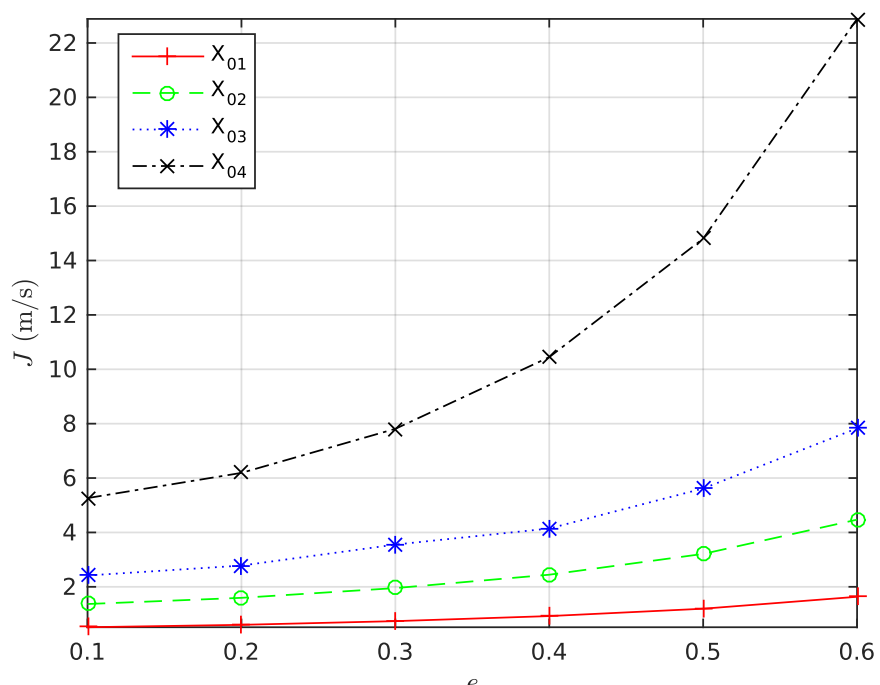

Figure 13: Impact of eccentricity $e$ on the fuel-consumption $J(\Delta V)$.

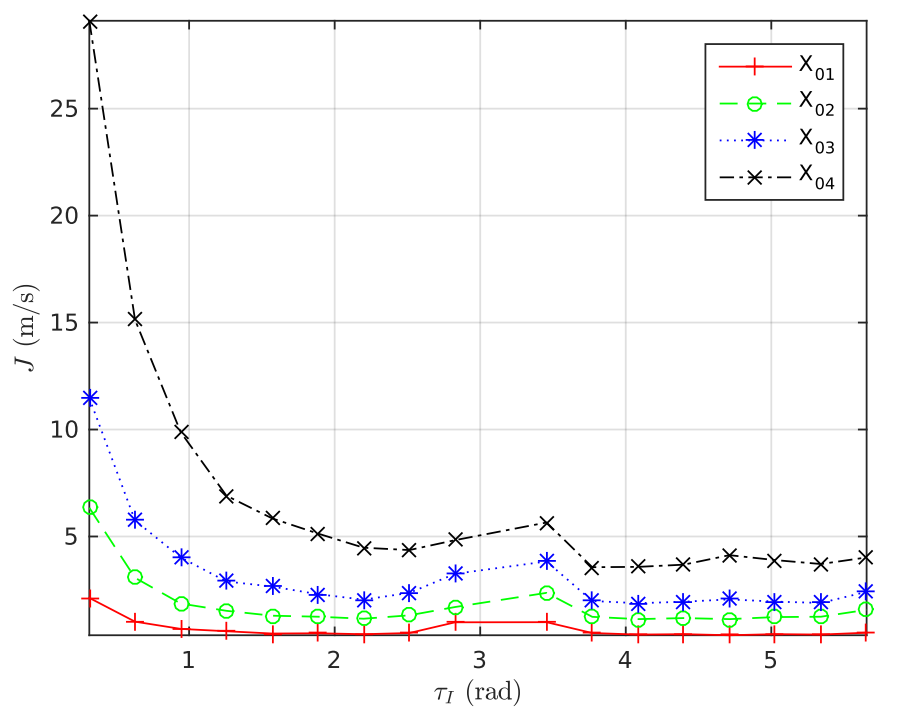

Figure 14: Impact of the interval $\tau_{I}$ on the fuel-consumption $J(\Delta V)$. 


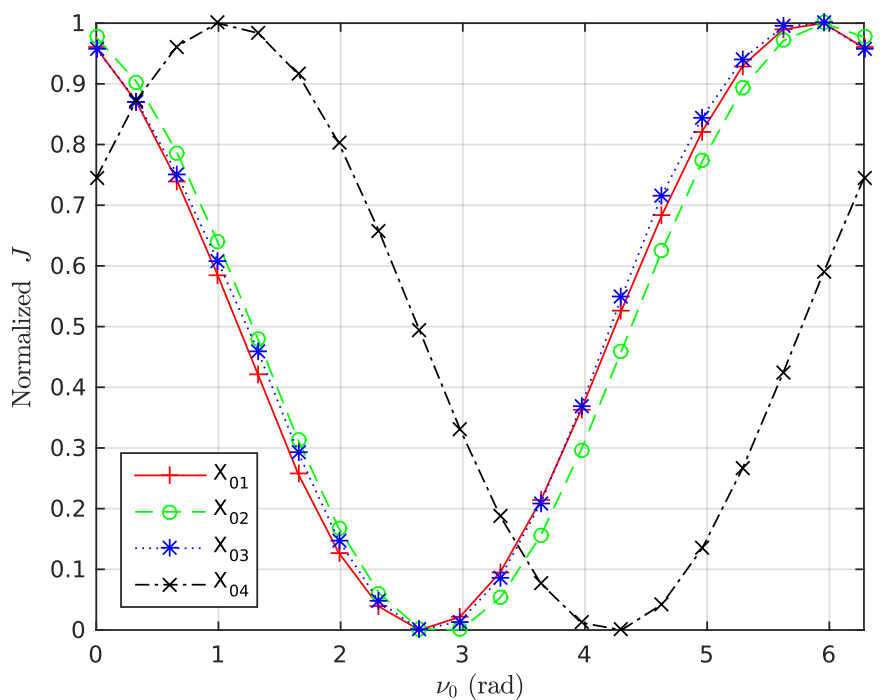

Figure 15: Impact of the interval initial true anomaly $\nu_{0}$ on the normalized fuel-consumption $J(\Delta V)$.

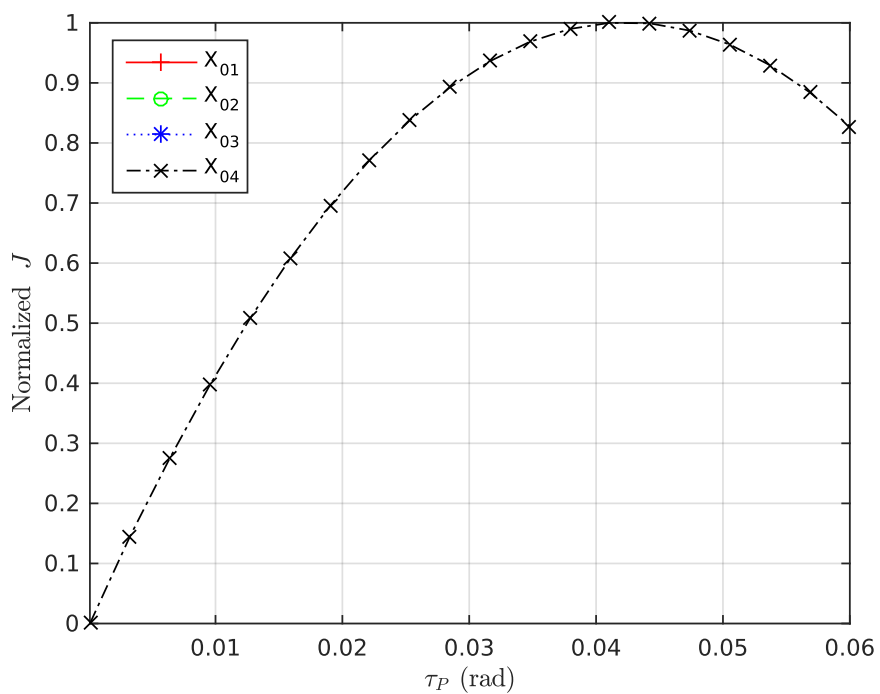

Figure 16: Impact of the interval $\tau_{p}$ on the normalized fuel-consumption $J(\Delta V)$

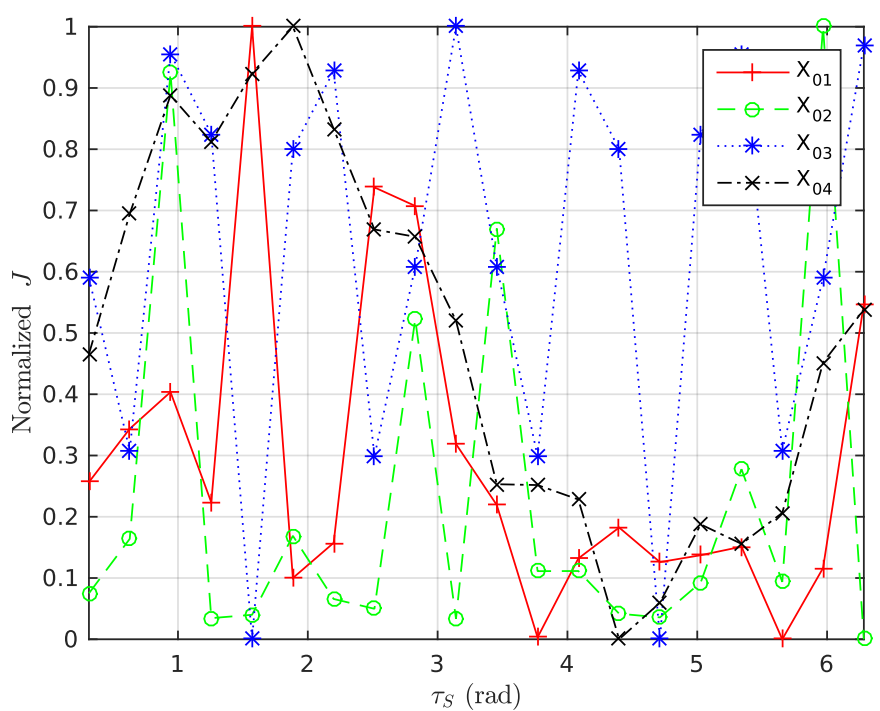

Figure 17: Impact of the interval $\tau_{S}$ on the normalized fuel-consumption $J(\Delta V)$. 
generating a periodic relative trajectory included in the hovering region.

Hardware-in-the-loop simulations using a LEON3 synthesized microprocessor reveal that although the proposed approach may produce greater convergence times, it is more efficient with respect to fuelconsumption than other methods proposed in the literature. Moreover, the timings obtained during these tests bring out the fact that this approach can be efficiently embedded in space dedicated devices. Finally, an analysis of the impact of the parameters rendezvous scenarios on the fuel-consumption is also presented.

Future works should focus in investigating the robustness of the proposed controller from a theoretical point of view, providing, for example, an idea of the influence of the nonlinearities, disturbances and scenario parameters on the stability of the method.

An extension of this work could combine safety requirements such as collision avoidance, passive safety or visibility, with our proposed station-keeping algorithm. This is due to the fact that existing guidance algorithms [35] which handle these constraints have the same mathematical formalism as the constrained optimization presented in this work. This can be done by considering time-varying path constraints, but this would imply revisiting the stability analysis while accounting for such time-varying constraints.

\section{Appendix A: Proofs}

\section{A.1 Proof of Proposition 2.1}

Proof. The convexity can be straightforwardly proven by showing that any convex combination of two elements of $S_{D}$ also belongs to $S_{D}$. In order to prove the boundedness of the set, we evaluate the inequalities in (23) at specific values:

Computing the bounds for $d_{1}$ and $d_{2}$ : by evaluating the $z$ inequalities at $\nu=0$ and $\nu=\frac{\pi}{2}$, we obtain:

$$
\begin{aligned}
& \underline{z} \leq d_{1} \leq \bar{z} \\
& \underline{z} \leq d_{2} \leq \bar{z}
\end{aligned}
$$

Computing the bounds for $d_{3}$ : by evaluating the $x$ inequalities at $\nu=0, \nu=\frac{2 \pi}{3}$ and $\nu=\frac{4 \pi}{3}$ and manipulating the produced inequalities, we obtain:

$$
\underline{x}\left(\frac{4+e-e^{2}}{4+e}\right) \leq d_{3} \leq \bar{x}\left(\frac{4+e-e^{2}}{4+e}\right)
$$


Computing the bounds for $d_{4}$ and $d_{5}$ : by evaluating the $y$ inequalities at $\nu=0$ and $\nu=\frac{\pi}{2}$, we obtain:

$$
\begin{gathered}
\underline{y}(1+e) \leq d_{4} \leq \bar{y}(1+e) \\
\underline{y} \leq d_{5} \leq \bar{y}
\end{gathered}
$$

Then, since any element of $S_{D}$ is entry-wise bounded, the set $S_{D}$ is itself bounded.

The closeness of $S_{D}$ can be proved by demonstrating that its boundary is contained in it. In order to do so, it suffices to show that the boundary of $S_{D}$ is the set of elements for which at least one of the inequalities in (23) is strictly satisfied for some $\nu$ :

$(\Rightarrow)$ Let $D$ be an element in the boundary of $S_{D}$. Then, for any $\varepsilon>0$, there exists a ball of radius $\varepsilon$ around $D$ containing elements that do not belong to $S_{D}$, i.e. elements for which at least one of the inequalities in (23) are not satisfied for some $\nu$. When the radius $\varepsilon$ of the ball around $D$ tends to zero, by continuity of the functions $F_{x}, F_{y}$ and $F_{z}$ on $\nu$ and $D$, the elements in this ball around $D$ tend to strictly satisfy at least one of the above cited inequalities for some $\nu$. This proves that, if $D$ belongs to the boundary of $S_{D}$, then it strictly satisfies at least one of the inequalities in (23) for some $\nu$.

$(\Leftarrow)$ Let $D$ be an element of $S_{D}$ for which at least one of the inequalities of $(23)$ is satisfied strictly for some $\nu^{*}$. Without any loss of generality, suppose that the vector $D$ satisfies:

$$
F_{z}\left(\nu^{*}\right) D=\cos \left(\nu^{*}\right) d_{1}+\sin \left(\nu^{*}\right) d_{2}=\bar{z}
$$

Now, consider the ball of radius $\varepsilon>0$ around $D$. For any value of $\varepsilon$, this ball contains elements that do not belong to $S_{D}$ (one example is the vector $D^{*}$ with same entries as $D$, except for $d_{1}^{*}=d_{1}+\operatorname{sgn}\left(\cos \left(\nu^{*}\right)\right) \varepsilon$ and $\left.d_{2}^{*}=d_{2}+\operatorname{sgn}\left(\sin \left(\nu^{*}\right)\right) \varepsilon\right)$. This proves that $D$ belongs to the boundary of $S_{D}$.

From the closeness and boundedness of $S_{D}$, we can conclude that it is also compact.

\section{A.2 Proof of Proposition 3.2}

Proof. The matrix $M_{y}\left(\nu_{1}, \ldots, \nu_{N}\right)$ has the following expression:

$$
M_{y}\left(\nu_{1}, \ldots, \nu_{N}\right)=k^{-2}\left[\begin{array}{rrr}
-\frac{\sin \left(\nu_{1}\right)}{\rho\left(\nu_{1}\right)} & \ldots & -\frac{\sin \left(\nu_{N}\right)}{\rho\left(\nu_{N}\right)} \\
\frac{\cos \left(\nu_{1}\right)}{\rho\left(\nu_{1}\right)} & \ldots & \frac{\cos \left(\nu_{N}\right)}{\rho\left(\nu_{N}\right)}
\end{array}\right]
$$

This matrix has rank 2 , since $\operatorname{det}\left(M_{y}\left(\nu_{1}, \nu_{2}\right)\right)=\frac{\sin \left(\nu_{2}-\nu_{1}\right)}{\rho\left(\nu_{1}\right) \rho\left(\nu_{2}\right)} \neq 0$ because of the hypothesis on $\nu_{1}, \ldots, \nu_{N}$. Then, $\forall D_{y}, D_{y}^{*} \in \mathbb{R}^{2}$ the vector $\Delta V_{y}=\left(M_{y}^{T} M_{y}\right)^{-1} M_{y}^{T}\left(D_{y}^{*}-D_{y}\right)$ is well-defined and satisfies $D_{y}^{*}=$ $D_{y}+M_{y}\left(\nu_{1}, \ldots, \nu_{N}\right) \Delta V_{y}$. This is also particularly true if $D_{y}^{*} \in S_{D_{y}} \neq \emptyset$.

Let us choose $\Delta V_{x z}$ as in (33). As demonstrated in Proposition 3.1 it is always possible to set $d_{0_{x z}}^{+}$ to any arbitrary value with a single impulse. Since $M_{0}^{\perp}\left(\nu_{1}, \ldots, \nu_{N}\right) \lambda$ has no influence on the first entry 
of $D_{x z}^{*}-D_{x z}$, we conclude that $\Delta V_{0}$ can be chosen to set the first entry of $D_{x z}^{*}-D_{x z}$ to any arbitrary value.

Now, let us demonstrate that it is always possible to choose $\lambda$ that allow us to set the other three entries of $D_{x z}^{*}-D_{x z}$ to any arbitrary values. Computing $M_{x z} M_{0}^{\perp}\left(\nu_{1}, \ldots, \nu_{N}\right)$, we obtain:

$$
M_{x z} M_{0}^{\perp}\left(\nu_{1}, \ldots, \nu_{N}\right)=\left(k^{6}\left(e^{2}-1\right)^{2}\right)^{-1}\left[\begin{array}{ccccccccc}
0 & 0 & 0 & 0 & 0 & \ldots & 0 & 0 \\
\frac{\sin \left(\nu_{1}\right)}{\rho\left(\nu_{1}\right)} & \star & \frac{\sin \left(\nu_{2}\right)}{\rho\left(\nu_{2}\right)} & \star & \frac{\sin \left(\nu_{3}\right)}{\rho\left(\nu_{3}\right)} & \ldots & \star & \frac{\sin \left(\nu_{N}\right)}{\rho\left(\nu_{N}\right)} \\
-\frac{\cos \left(\nu_{1}\right)}{\rho\left(\nu_{1}\right)} & \star & -\frac{\cos \left(\nu_{2}\right)}{\rho\left(\nu_{2}\right)} & \star & -\frac{\cos \left(\nu_{3}\right)}{\rho\left(\nu_{3}\right)} & \ldots & \star & -\frac{\cos \left(\nu_{N}\right)}{\rho\left(\nu_{N}\right)} \\
\frac{1+\rho\left(\nu_{1}\right)}{\rho\left(\nu_{1}\right)} & \star & \frac{1+\rho\left(\nu_{2}\right)}{\rho\left(\nu_{2}\right)} & \star & \frac{1+\rho\left(\nu_{3}\right)}{\rho\left(\nu_{3}\right)} & \ldots & \star & \frac{1+\rho\left(\nu_{N}\right)}{\rho\left(\nu_{N}\right)}
\end{array}\right] .
$$

and this matrix has rank 3, since:

$$
\begin{aligned}
\operatorname{det}\left(\left[\begin{array}{ccc}
\frac{\sin \left(\nu_{1}\right)}{\rho\left(\nu_{1}\right)} & \frac{\sin \left(\nu_{2}\right)}{\rho\left(\nu_{2}\right)} & \frac{\sin \left(\nu_{3}\right)}{\rho\left(\nu_{3}\right)} \\
-\frac{\cos \left(\nu_{1}\right)}{\rho\left(\nu_{1}\right)} & -\frac{\cos \left(\nu_{2}\right)}{\rho\left(\nu_{2}\right)} & -\frac{\cos \left(\nu_{3}\right)}{\rho\left(\nu_{3}\right)} \\
\frac{1+\rho\left(\nu_{1}\right)}{\rho\left(\nu_{1}\right)} & \frac{1+\rho\left(\nu_{2}\right)}{\rho\left(\nu_{2}\right)} & \frac{1+\rho\left(\nu_{3}\right)}{\rho\left(\nu_{3}\right)}
\end{array}\right]\right) & =-2 \frac{\sin \left(\nu_{2}-\nu_{1}\right)+\sin \left(\nu_{3}-\nu_{2}\right)-\sin \left(\nu_{3}-\nu_{1}\right)}{k^{18}\left(e^{2}-1\right)^{6} \rho\left(\nu_{1}\right) \rho\left(\nu_{2}\right) \rho\left(\nu_{3}\right)} \\
& =-8 \frac{\sin \frac{\nu_{2}-\nu_{1}}{2} \sin \frac{\nu_{3}-\nu_{2}}{2} \sin \frac{\nu_{3}-\nu_{1}}{2}}{k^{18}\left(e^{2}-1\right)^{6} \rho\left(\nu_{1}\right) \rho\left(\nu_{2}\right) \rho\left(\nu_{3}\right)} \neq 0,
\end{aligned}
$$

because of the hypothesis on $\nu_{1}, \ldots, \nu_{N}$.

This implies that $\forall D_{x z}, D_{x z}^{*} \in \mathbb{R}^{4}$ it is possible to chose $\lambda$ and $\Delta V_{0}$ in such a manner that $\Delta V_{x z}(\lambda)=$ $M_{0}^{\perp}\left(\nu_{1}, \ldots, \nu_{N}\right) \lambda+\Delta V_{0}$ satisfies $D_{x z}^{*}=D_{x z}+M_{x z}\left(\nu_{1}, \ldots, \nu_{N}\right) \Delta V_{x z}$. This is also particularly true if $D_{x z}^{*} \in S_{D_{x z}} \neq \emptyset$.

\section{A.3 Proof of Proposition 3.5}

In order to prove Prop. 3.5, we introduce the following preliminary result:

Proposition A.1. Let $\mathbb{K} \subset \mathbb{R}^{n}$ be a convex set, $A, C \in \mathbb{R}^{n}, A \notin \mathbb{K}$ and $C \in \mathbb{K}$. Let $B$ be the projection of $A$ onto $\mathbb{K}$, defined as $B=\underset{\mathbb{K}}{\operatorname{proj}} A:=\underset{X \in \mathbb{K}}{\operatorname{argmin}}\|X-A\|_{2}$. Consider also the points $A^{\prime}=C+\lambda(A-C)$ for $0<\lambda<1$ and $B^{\prime}$ the projection of $A^{\prime}$ onto $\mathbb{K}$. Then, the distance between $A^{\prime}$ and $\mathbb{K}$ is less than the distance between $A$ and $\mathbb{K}$ i.e., $\inf _{X \in \mathbb{K}}\left\|X-A^{\prime}\right\|_{2}<\inf _{X \in \mathbb{K}}\|X-A\|_{2} . \quad\left(\operatorname{dist}_{\mathbb{K}} A^{\prime}:=\inf _{X \in \mathbb{K}}\left\|X-A^{\prime}\right\|_{2}<\operatorname{dist}_{\mathbb{K}} A:=\right.$ $\left.\inf _{X \in \mathbb{K}}\|X-A\|_{2}\right)$.

Proof. Consider the point $B^{\prime \prime}=C+\lambda(B-C)$ (see Figure 18). Since $\mathbb{K}$ is convex and $B^{\prime \prime}$ belongs to the segment $\overline{B C}, B^{\prime \prime}$ is a point of $\mathbb{K}$. By developing the difference $B^{\prime \prime}-A^{\prime}$ :

$$
B^{\prime \prime}-A^{\prime}=\lambda(B-A) \Rightarrow\left\|B^{\prime \prime}-A^{\prime}\right\|_{2}=\lambda\|B-A\|_{2} .
$$




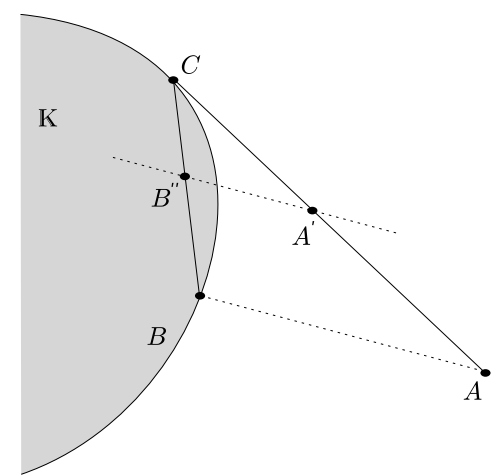

Figure 18: Illustration of points $A, A^{\prime}, B, B^{\prime \prime}, C$ and convex $\mathbb{K}$

But since $B^{\prime}$ is the projection of $A^{\prime}$ onto $\mathbb{K}$ :

$$
\left\|B^{\prime}-A^{\prime}\right\|_{2} \leq\left\|B^{\prime \prime}-A^{\prime}\right\|_{2}=\lambda\|B-A\|_{2} \Rightarrow\left\|B^{\prime}-A^{\prime}\right\|_{2}<\|B-A\|_{2} .
$$

Now we proceed with the proof of Prop. 3.5:

Proof. From Prop. 3.2, the functions $\gamma_{y}$ and $\gamma_{x z}$ returns a sequence of impulses that generates an admissible trajectory, i.e. $\phi_{k}=\phi_{k-1}+M\left(\nu_{k}^{(1)}, \ldots, \nu_{k}^{(N)}\right) \Delta V_{k} \in S_{D}$. Then, if for some $k^{*}$ the impulse $\Delta V_{k^{*}}$ respects the saturation constraint, we have that $\forall k \geq k^{*}, \phi_{k} \in S_{D}$.

However, suppose that the saturation is always violated for any $k \in \mathbb{N}$ (worst case scenario). By writing the expressions of $\phi_{k}$ considering the non-scaled and the scaled sequence of impulses, we obtain:

$$
\begin{aligned}
& \bar{\phi}_{k}=\phi_{k-1}+M\left(\nu_{k}^{(1)}, \ldots, \nu_{k}^{(N)}\right) \Delta V_{k} \\
& \phi_{k}=\phi_{k-1}+M\left(\nu_{k}^{(1)}, \ldots, \nu_{k}^{(N)}\right) \Delta V_{k} \overline{\Delta V} /\left\|\Delta V_{k}\right\|_{\infty}
\end{aligned}
$$

By manipulating the previous equations, we obtain the following expression:

$$
\bar{\phi}_{k}-\phi_{k}=\left(1-\overline{\Delta V} /\left\|\Delta V_{k}\right\|_{\infty}\right)\left(\bar{\phi}_{k}-\phi_{k-1}\right)
$$

From the saturation hypothesis we have that $\overline{\Delta V}<\left\|\Delta V_{k}\right\|_{\infty}$ and consequently:

$$
\left\|\bar{\phi}_{k}-\phi_{k}\right\|_{2}=\left(1-\overline{\Delta V} /\left\|\Delta V_{k}\right\|_{\infty}\right)\left\|\bar{\phi}_{k}-\phi_{k-1}\right\|_{2}<\left\|\bar{\phi}_{k}-\phi_{k-1}\right\|_{2}
$$

Since $\bar{\phi}_{k}$ belongs to $S_{D}$, which is a convex set, from Prop. A.1 we conclude that:

$$
\operatorname{dist}_{S_{D}}\left(\phi_{k}\right) \leq\left(1-\overline{\Delta V} /\left\|\Delta V_{k}\right\|_{\infty}\right) \operatorname{dist}_{S_{D}}\left(\phi_{k-1}\right)<\operatorname{dist}_{S_{D}}\left(\phi_{k-1}\right)
$$


We then define the following sets:

$$
\begin{gathered}
P_{k}:=\left\{D \in \mathbb{R}^{2} \mid \operatorname{dist}(D) \leq \operatorname{disst}_{S_{D}}\left(\phi_{k-1}\right)\right\}, \\
Q_{y_{k}}:=\left\{\Delta V_{y} \in \mathbb{R}^{2} \mid \exists \nu \in \mathbb{R}, \exists D \in P_{k} \text { s.t. } \Delta V_{y}=\gamma_{y}\left(D, \nu, \ldots, \nu+(N-1) \tau_{I}\right)\right\}, \\
Q_{x z_{k}}:=\left\{\Delta V_{x z} \in \mathbb{R}^{2} \mid \exists \nu \in \mathbb{R}, \exists D \in P_{k} \text { s.t. } \Delta V_{x z}=\gamma_{x z}\left(D, \nu, \ldots, \nu+(N-1) \tau_{I}\right)\right\},
\end{gathered}
$$

and $\Delta V_{k}^{\star}:=\max \left\{\max _{\Delta V_{y} \in Q_{y_{k}}}\left\|\Delta V_{y}\right\|_{\infty} \max _{\Delta V_{x z} \in Q_{x z_{k}}}\left\|\Delta V_{x z}\right\|_{\infty}\right\}$. Since $\operatorname{dist}_{S_{D}}\left(\phi_{k}\right)<\operatorname{dist}\left(\phi_{k-1}\right)$, the sets $Q_{k}$ form a sequence of inclusions $Q_{k+1} \subseteq Q_{k}$ and, consequently, $\Delta V_{k+1}^{\star} \leq \Delta V_{k}^{\star}$. Now let us define the following two sequences:

$$
\left(a_{k}\right)_{k \in \mathbb{N}}:=\left\{\begin{array}{l}
a_{0}=\operatorname{dist}_{S_{D}}\left(\phi_{0}\right), \\
a_{k}=\alpha a_{k-1}
\end{array} \quad \text { and } \quad\left(b_{k}\right)_{k \in \mathbb{N}}:=\left\{\begin{array}{l}
b_{0}=\operatorname{dist}_{S_{D}}\left(\phi_{0}\right) \\
b_{k}=\operatorname{dist}_{S_{D}}\left(\phi_{k-1}\right)
\end{array}\right.\right.
$$

where $\alpha=\left(1-\overline{\Delta V} / \Delta V_{1}^{\star}\right)$. The sequence $\left(a_{k}\right)_{k \in \mathbb{N}}$ has a general term of the form $a_{k}=\alpha^{k} a_{0}$ and converges to zero when $k$ tends to infinite: $0<\alpha<1 \Rightarrow a_{k} \underset{k \rightarrow \infty}{\rightarrow} 0$. The second sequence represents the distance of the terms of the sequence $\phi_{k}$ to the admissible set $S_{D}$. Since we suppose that the saturation is always violated, we have the following inequalities:

$$
\overline{\Delta V}<\left\|\Delta V_{k}\right\|_{\infty}<\Delta V_{k}^{\star} \leq \Delta V_{1}^{\star}, \quad \forall k \in \mathbb{N}
$$

Then, since $\operatorname{dist}_{S_{D}}\left(\phi_{k}\right) \leq\left(1-\overline{\Delta V} /\left\|\Delta V_{k}\right\|_{\infty}\right) \operatorname{dist}_{S_{D}}\left(\phi_{k-1}\right)$ and $\forall k \in \mathbb{N},\left(1-\overline{\Delta V} /\left\|\Delta V_{k}\right\|_{\infty}\right)<\alpha$, by comparing the sequences $\left(a_{k}\right)_{k \in \mathbb{N}}$ and $\left(b_{k}\right)_{k \in \mathbb{N}}$ we prove that $b_{k} \underset{k \rightarrow \infty}{\rightarrow} 0$, which is equivalent to $\operatorname{dist}_{S_{D}}\left(\phi_{k}\right) \underset{k \rightarrow \infty}{\rightarrow} 0$.

\section{Acknowledgments}

This work was supported by the FastRelax (ANR-14-CE25-0018-01) project of the French National Agency for Research (ANR).

\section{References}

[1] D. J. Irvin, R. G Cobb, and T. A. Lovell. Fuel-optimal maneuvers for constrained relative satellite orbits. Journal of guidance, control, and dynamics, 32(3):960-973, 2009. doi: 10.2514/1.36618.

[2] D. Bainov and P. Simeonov. Impulsive differential equations: periodic solutions and applications, volume 66. CRC Press, 1993. ISBN 9780582096394.

[3] C. Andrade, R. Ramirez-Mendoza, M. Giacoman-Zarzar, R. Morales, A. Fejzic, A. Saenz-Otero, and D. W. Miller. 
Robust control applied towards rendezvous and docking. In 2009 European Control Conference (ECC), pages 18541859, Aug 2009. doi: 10.23919/ECC.2009.7074673.

[4] P. Singla, K. Subbarao, and J. L. Junkins. Adaptive output feedback control for spacecraft rendezvous and docking under measurement uncertainty. Journal of Guidance, Control, and Dynamics, 29(4):892-902, jul 2006. doi: 10.2514/ 1.17498 .

[5] H. Schaub and K. T. Alfriend. Impulsive feedback control to establish specific mean orbit elements of spacecraft formations. Journal of Guidance, Control, and Dynamics, 24(4):739-745, jul 2001. doi: 10.2514/2.4774.

[6] Q. Yan, G. Yang, V. Kapila, and M.S. de Queiroz. Nonlinear dynamics and output feedback control of multiple spacecraft in elliptical orbits. In Proceedings of the 2000 American Control Conference. ACC. IEEE, 2000. doi: $10.1109 /$ acc. 2000.876617 .

[7] W. M. Haddad, V. Chellaboina, and S. G. Nersesov. Impulsive and hybrid dynamical systems. Princeton Series in Applied Mathematics. Princeton, 2006.

[8] E. N. Hartley. A tutorial on model predictive control for spacecraft rendezvous. In 2015 European Control Conference (ECC), pages 1355-1361, July 2015. doi: 10.1109/ECC.2015.7330727.

[9] E. Capello, F. Dabbene, G. Guglieri, and E. Punta. "Flyable" Guidance and Control Algorithms for Orbital Rendezvous Maneuver. SICE Journal of Control, Measurement, and System Integration, 11(1):14-24, 2018. doi: 10.9746/jcmsi.11.14.

[10] G. Gaias and S. D'Amico. Impulsive maneuvers for formation reconfiguration using relative orbital elements. Journal of Guidance, Control, and Dynamics, 38(6):1036-1049, April 2014. doi: 10.2514/1.G000189.

[11] G. Gaias, S. D'Amico, and J-S. Ardaens. Generalised multi-impulsive manoeuvres for optimum spacecraft rendezvous in near-circular orbit. International Journal of Space Science and Engineering, 3(1):68-88, 2015. doi: 10.1504/ IJSPACESE.2015.069361.

[12] A. Robertson, G. Inalhan, and J. P. How. Formation control strategies for a separated spacecraft interferometer. In Proceedings of the 1999 American Control Conference, volume 6, pages 4142-4147, June 1999. doi: 10.1109/ACC. 1999.786331.

[13] M. Tillerson, G. Inalhan, and J. P. How. Co-ordination and control of distributed spacecraft systems using convex optimization techniques. International Journal of Robust and Nonlinear Control, 12(2-3):207-242, 2002. doi: 10. $1002 /$ rnc.683.

[14] K. Yamanaka and F. Ankersen. New state transition matrix for relative motion on an arbitrary elliptical orbit. Journal of Guidance, Control, and Dynamics, 25(1):60-66, jan 2002. doi: 10.2514/2.4875.

[15] G. Inalhan, M. Tillerson, and J. P. How. Relative dynamics and control of spacecraft formations in eccentric orbits. Journal of Guidance, Control, and Dynamics, 25(1):48-59, January 2002. doi: 10.2514/2.4874.

[16] L. S. Breger and J. P. How. Safe trajectories for autonomous rendezvous of spacecraft. Journal of Guidance, Control, and Dynamics, 31(5):1478-1489, 2008. doi: 10.2514/6.2006-6584.

[17] A. Richards, T. Schouwenaars, J. P. How, and E. Feron. Spacecraft trajectory planning with avoidance constraints using mixed-integer linear programming. Journal of Guidance, Control, and Dynamics, 25(4):755-764, July 2002. doi: $10.2514 / 2.4943$. 
[18] J. B. Mueller and R. Larsson. Collision avoidance maneuver planning with robust optimization. In International ESA Conference on Guidance, Navigation and Control Systems, Tralee, County Kerry, Ireland, 2008.

[19] J. P. How and M. Tillerson. Analysis of the impact of sensor noise on formation flying control. In Proceedings of the 2001 American Control Conference, volume 5, pages 3986-3991 vol.5, June 2001. doi: 10.1109/ACC.2001.946298.

[20] C. Louembet, D. Arzelier, and G. Deaconu. Robust rendezvous planning under maneuver execution errors. Journal of Guidance, Control, and Dynamics, 38(1):76-93, October 2015. doi: 10.2514/1.G000391.

[21] S. Di Cairano, H. Park, and I. Kolmanovsky. Model predictive control approach for guidance of spacecraft rendezvous and proximity maneuvering. International Journal of Robust and Nonlinear Control, 22(12):1398-1427, 2012. doi: $10.1002 /$ rnc. 2827 .

[22] H. Park, C. Zagaris, J. Virgili Llop, R. Zappulla, I. Kolmanovsky, and M. Romano. Analysis and experimentation of model predictive control for spacecraft rendezvous and proximity operations with multiple obstacle avoidance. In AIAA/AAS Astrodynamics Specialist Conference. American Institute of Aeronautics and Astronautics, sep 2016. doi: $10.2514 / 6.2016-5273$.

[23] L. S. Breger, G. Inalhan, M. Tillerson, and J. P. How. Cooperative Spacecraft Formation Flying: Model Predictive Control with Open- and Closed-Loop Robustness, volume 1 of Elsevier Astrodynamics Series, pages 237-277. Butterworth-Heinemann, 2006. doi: 10.1016/s1874-9305(07)80010-0.

[24] F. Gavilan, R. Vazquez, and E. F. Camacho. Chance-constrained model predictive control for spacecraft rendezvous with disturbance estimation. Control Engineering Practice, 20(2):111-122, 2012. doi: 10.1016/j.conengprac.2011.09. 006.

[25] F. Lobo Pereira, F. A. C. C. Fontes, A. Pedro Aguiar, and J. Borges de Sousa. An Optimization-Based Framework for Impulsive Control Systems, pages 277-300. Springer International Publishing, 2015. ISBN 978-3-319-26687-9. doi: 10.1007/978-3-319-26687-9_13.

[26] A. Richards and J. P. How. Robust variable horizon model predictive control for vehicle maneuvering. International Journal of Robust and Nonlinear Control, 16(7):333-351, 2006. doi: 10.1002/rnc.1059.

[27] Y. Kuwata, A. Richards, and J. How. Robust receding horizon control using generalized constraint tightening. In 2007 American Control Conference, pages 4482-4487, July 2007. doi: 10.1109/ACC.2007.4283000.

[28] R. C. Shekhar and J. M. Maciejowski. Optimal constraint tightening policies for robust variable horizon model predictive control. In 51st IEEE Conference on Decision and Control (CDC), pages 5170-5175, December 2012. doi: 10.1109/CDC.2012.6426710.

[29] G. Deaconu, C. Louembet, and A. Théron. Minimizing the effects of navigation uncertainties on the spacecraft rendezvous precision. Journal of Guidance, Control, and Dynamics, 37(2):695-700, February 2014. doi: 10.2514/1. 62219.

[30] D. Limon, I. Alvarado, T. Alamo, and E. F. Camacho. Robust tube-based mpc for tracking of constrained linear systems with additive disturbances. Journal of Process Control, 20(3):248-260, 2010. doi: 10.1016/j.jprocont.2009. 11.007 .

[31] D. Q. Mayne, E. C. Kerrigan, and P. Falugi. Robust model predictive control: advantages and disadvantages of tubebased methods. In 18th IFAC World Congress, volume 44, pages 191-196, 2011. doi: 10.3182/20110828-6-IT-1002. 01893. 
[32] W. Langson, I. Chryssochoos, S.V. Raković, and D.Q. Mayne. Robust model predictive control using tubes. Automatica, 40(1):125-133, 2004. doi: 10.1016/j.automatica.2003.08.009.

[33] R.S. Wiltshire W.H. Clohessy. Terminal guidance system for satellite rendezvous. Journal of the Aerospace Sciences, 27(9):653-658, sep 1960. doi: 10.2514/8.8704. URL https://doi.org/10.2514\%2F8.8704.

[34] J. Tschauner. Elliptic orbit rendezvous. AIAA Journal, 5(6):1110-1113, 1967. doi: 10.2514/3.4145.

[35] G. Deaconu, C. Louembet, and A. Théron. Designing continuously constrained spacecraft relative trajectories for proximity operations. Journal of Guidance, Control, and Dynamics, 38(7):1208-1217, 2015. doi: 10.1109/acc.2012. 6314659.

[36] P. R. Arantes Gilz, M. Joldes, C. Louembet, and F. Camps. Model predictive control for rendezvous hovering phases based on a novel description of constrained trajectories. In 20th IFAC World Congress, volume 50, pages 7229-7234, 2017. doi: 10.1016/j.ifacol.2017.08.1367.

[37] A. Ferramosca P. S. Rivadeneira and A. H. González. MPC with State Window Target Control in Linear Impulsive Systems. IFAC-PapersOnLine, 48(23):507-512, 2015. doi: 10.1016/j.ifacol.2015.11.329. 5th IFAC Conference on Nonlinear Model Predictive Control NMPC 2015.

[38] M. Brentari, S. Urbina, D. Arzelier, C. Louembet, and L. Zaccarian. A hybrid control framework for impulsive control of satellite rendezvous. IEEE Transactions on Control Systems Technology, pages 1-15, 2018. doi: 10.1109/tcst.2018. 2812197.

[39] E. N. Hartley and J. M. Maciejowski. Predictive control for spacecraft rendezvous in an elliptical orbit using an FPGA. In Control Conference (ECC), 2013 European, pages 1359-1364. IEEE, 2013. doi: 10.23919/ECC.2013.6669196.

[40] E. N. Hartley and J. M. Maciejowski. Field programmable gate array based predictive control system for spacecraft rendezvous in elliptical orbits. Optimal Control Applications and Methods, 36(5):585-607, 2015. doi: 10.1002/oca.2117.

[41] P. R. Arantes Gilz. A Matlab®/Simulink® non-linear simulator for orbital spacecraft rendezvous applications., December 2016. URL https://hal.archives-ouvertes.fr/hal-01413328.

[42] F. Camps, P. R. Arantes Gilz, M. Joldes, and C. Louembet. Embedding a SDP-based control algorithm for the orbital rendezvous hovering phases. In 2018 25th Saint Petersburg International Conference on Integrated Navigation Systems (ICINS). IEEE, may 2018. doi: 10.23919/icins.2018.8405931.

[43] G. Deaconu. On the trajectory design, guidance and control for spacecraft rendezvous and proximity operations. PhD thesis, Univ. Toulouse 3 - Paul Sabatier, Toulouse, France, October 2013.

[44] Y. Nesterov. Squared functional systems and optimization problems. In T. Terlaky H. Frenk, K. Roos and S. Zhang, editors, High Performance Optimization. Applied Optimization, volume 33, pages 405-440. Springer US, 2000. doi: 10.1007/978-1-4757-3216-0_17.

[45] B. Borchers. CSDP, A C library for semidefinite programming. Optimization Methods and Software, 11(1-4):613-623, 1999. doi: 10.1080/10556789908805765.

[46] A. S. Lewis and M. L. Overton. Nonsmooth optimization via quasi-Newton methods. Mathematical Programming, 141(1-2):135-163, Oct 2013. doi: 10.1007/s10107-012-0514-2.

[47] N. Z. Shor, K. C. Kiwiel, and A. Ruszcayǹski. Minimization methods for non-differentiable functions, volume 3. Springer-Verlag, Berlin, Heidelberg, 1985. ISBN 0-387-12763-1. doi: 10.1007/978-3-642-82118-9. 
[48] PENDER. GR-XC6S Development Board - User Manual, July 2011. URL https://goo.gl/Snt6vj. Accessed Nov. $17,2017$.

[49] ESA. Onboard computer and data handling, May 2014. URL https://goo.gl/1PZNkC. Accessed Nov. $17,2017$.

[50] D. A. Vallado. Fundamentals of astrodynamics and applications, volume 12. Springer, 2001. ISBN 9781881883197.

[51] M. J. H. Walker. A set of modified equinoctial orbit elements. Celestial mechanics, 38(4):391-392, 1986. doi: $10.1007 /$ bf01238929.

[52] P. Bodin, R. Larsson, F. Nilsson, C. Chasset, R. Noteborn, and M. Nylund. PRISMA: An in-orbit test bed for guidance, navigation, and control experiments. Journal of Spacecraft and Rockets, 46(3):615-623, 5 2009. doi: 10.2514/1.40161. 